\title{
Exploiting social features for improving cognitive radio infrastructures and social services via combined MRF and back pressure cross-layer resource allocation
}

\author{
Evangelos Anifantis ${ }^{*}{ }^{\dagger}$, Eleni Stai ${ }^{\dagger}$, Vasileios Karyotis and Symeon Papavassiliou
}

\author{
*Correspondence: \\ vangelis@netmode.ntua.gr \\ †Equal contributors \\ School of Electrical and Computer \\ Engineering, National Technical \\ University of Athens (NTUA), 15780 \\ Zografou, Athens, Greece
}

\begin{abstract}
The observed unbalance of spectrum utilization combined with the proliferation of wireless devices and the growth of online social networks call for a joint study of cognitive radios (CRs) and social networking towards improving the operation of both the underlying CR-capable communications infrastructures and of the corresponding dynamic networks. In this work, we address such issue and focus on the twofold direction of resource allocation in CRs and social feature exploitation with the aim of providing a holistic cross-layer optimization framework that jointly considers information from the social layer as well as the traditional network protocol layers. We combine Markov random field (MRF) cross-layer decisions with back pressure (BP) features for the allocation of resources, in order to improve the operation of CRs that act as social network substrate. Espousing the recent advances in the field of dynamic spectrum allocation, the proposed BP-enhanced MRF (BPeMRF) network optimization serves the purpose of improving in a low complexity manner the capacity of agile infrastructures, which are required to adapt fast according to the demands of the volatile CR environment and the overlaying dynamic online social networks. In addition, within the proposed socially enhanced BPeMRF (SeBPeMRF) approach, we leverage emerging social information to further enhance performance. The obtained results exhibit the efficacy of BPeMRF/SeBPeMRF regarding the above objectives and demonstrate significant promise for further improving the corresponding infrastructures and services.
\end{abstract}

Keywords: Cognitive radio networks; Social features; Markov random fields; Back pressure; Cross-layer design; Network enhancement

\section{Introduction}

Lately, various studies [1,2] have proved the significant unbalance of spectrum assignment and utilization generated by the traditional static spectrum allocation practices worldwide. Simultaneously, wireless technology and online social networks have experienced tremendous growth, so that nowadays, most of the Internet access takes place through smart handhelds [3]. In addition, wireless data traffic increases considerably, mainly due to the widespread proliferation of online social networks and the associated offered

(c) 2014 Anifantis et al.; licensee Springer. This is an Open Access article distributed under the terms of the Creative Commons 
services/applications [4]. Such trends have motivated the design of more intelligent wireless networks, e.g., cognitive radios (CRs), and are expected to leverage diverse information, while also shape the evolution of the future wireless Internet.

Social networking on its part, i.e., online social networks and mobile social networks [5], has started shaping the current and future trends of wireless traffic, and thus, network engineers have to take into account their features, complications, and other requirements they impose on the operation of networks. In general, it seems that CRs and social networking are becoming more integrated in the future wireless Internet, in various capacities, and they seem to converge in the sense that CRs may constitute one of the major infrastructures to carry online social network traffic and meet the corresponding demands imposed by the latter. In this work, we take this approach one step ahead, and in addition to demonstrating how one can achieve the above, we will also show that features and additional information from the social network layer can be exploited to further improve the underlying CR infrastructure. In other words, this paper will contribute towards creating a promising bond between CRs and social networking for the future wireless Internet.

The CR paradigm [6] has been conceived in a brain-empowered manner to address the spectrum underutilization problem and provide spectrum-agile communications, following a methodology that mimics the human behavior and by extension the evolutionary processes of physical, biological, and other systems that exhibit social structure and cognition [7]. Their applications include - among others - big social events such as conferences, sports finals, etc., where traffic bottlenecks arise quickly when many users attempt to access simultaneously scarce resources, e.g., available channels, in a static/inefficient manner. Above all, CRs can be used to alleviate problems emerging in cyber-physical systems combining wireless infrastructures and online social networks, e.g., to provide on-the-fly traffic offloading of the cellular infrastructure, thus expediting data transfers and reducing delays (LTE advanced) in crowded locations. At the same time, the cognition-based operation of CRs requires dynamic reconfigurations and flexible adaptations in order to conform to higher priority user requirements and support efficiently the anticipated quality of service (QoS) demands by the future services and applications developing in diverse wireless and dynamic online social networks.

Based on the aforementioned observations/emerging trends, we focus on the design and analysis of spectrum-agile communications networks, aiming at supporting the increased QoS requirements imposed by current and future dynamic and online social networks. We propose a network optimization approach for CR networks, which aims at jointly determining the transmission power and channel allocation while achieving optimal scheduling and routing through lightweight and distributed computation. The optimization proposed by our approach is based on a Markov random field (MRF) [8] cross-layer formulation and the inherently distributed Gibbs sampling technique [9] enhanced with back pressure (BP) [10] features, with the goal of increasing capacity, avoiding interference, and supporting the development of flexible spectrum-agile infrastructures for dynamic online social networks.

More specifically, the proposed BP-enhanced MRF (BPeMRF) approach requires the minimum possible information from a centralized spectrum database and it can proceed with distributed computation in order to determine the transmission power and channel allocation through an MRF decision model. BPeMRF exploits BP features for ensuring 
optimal scheduling and determining routing at the higher protocol stack layers. We compare the joint mechanism with a traditional BP scheme in order to validate its efficiency and performance. The simulation results follow the lines of the theoretical results, indicating the throughput optimal performance of the BPeMRF scheme, similarly to BP, while by leveraging Gibbs sampling, BPeMRF allows for less complex computations than BP.

In addition, we further enhance the BPeMRF algorithm with information available from the social layer, proposing the socially enhanced BPeMRF (SeBPeMRF) algorithm. This approach addresses the bidirectional interplay between resource allocation in CRs and social layer's operation that seems to offer significant benefits in exploiting social features to enhance the infrastructure adaptively to both the network protocol layers and the social layer. We demonstrate the efficacy of such approach via two use cases in the 'Case study I: reputation of PUs appearance' and 'Case study II: adapting routing efficiency to social layer's needs' sections.

The rest of this paper is structured as follows. In the 'Background and contributions' section, we describe relevant works and highlight the contributions of our work, while in the 'System model' section, we explain the assumed system model. The 'MRF-based resource allocation framework for centralized CRNs' section introduces the design of BPeMRF approach by presenting the MRF part as well as the BP features of BPeMRF. In the 'Exploiting social information in CRN cross-layer optimization and resource allocation' section, the incorporation of social awareness via SeBPeMRF approach is analyzed, while performance evaluation of the proposed BPeMRF and simulation results via two case studies of the proposed SeBPeMRF are provided in the 'Simulation results' section. Finally, the 'Conclusion' section concludes the paper.

\section{Background and contributions}

CRs received considerable attention the last decade, towards providing a flexible communications paradigm. In response to the immature purely ad hoc CR technology, regulation bodies have already promoted viable infrastructure-based cognitive radio networks (CRNs) assisted by centralized spectrum databases, e.g., [11]. Our work aligns with this centralized framework and aims at further improving SU operation. Various crosslayer analyses of centralized CRNs have emerged in the literature [12-15], and compared to them, our work further introduces an MRF-based cross-layered resource allocation approach in a suitable manner for incorporating social information. MRF formulation serves for modeling spatial dependencies and, along with Gibbs sampling, has attracted the attention of diverse research fields due to the inherent capability for addressing a class of problems with large space of possible configurations. Gibbs sampling accompanied by MRFs has been initially proposed for image restoration in [9], and it was recently employed on resource allocation problems in wireless networks [16-18]. In this paper, we focus on leveraging MRF structure and Gibbs sampling combined with back pressure features such that throughput optimal joint scheduling and routing is efficiently achieved along with channel allocation and power control in the secondary network.

The traditional back pressure scheduling/routing algorithm, initially proposed in [10], ensures throughput optimality based on congestion gradients, while solving an NP-hard centralized maximum weight matching (MWM) problem [19], with complexity depending on the number of maximal independent sets of the underlying network graph. To overcome this, several suboptimal schemes have been developed to approximate the 
solution of the MWM [19]. Greedy maximal scheduling (GMS) (longest queue first) is an alternative that maintains performance close to optimal while being throughput optimal for special topologies and can be replaced equivalently by local GMS which uses only local information [19]. Also, [20] presents a broad category of alternative approaches based on randomized schedulers that allow for imperfections and relaxations on their operation. The introduction of imperfections can be exploited for the development of low complexity (e.g., polynomial) and distributed schedulers under a general interference model. Contrary to the above works approximating MWM, our approach achieves throughput optimality with low-complexity and local information exchange, by avoiding solving MWM and relying on a converged Gibbs sampler for obtaining the optimal schedule at each time slot.

The proposed replacement of the MWM facilitating the applicability of the back pressure algorithm, along with the efficient spectrum utilization achieved via CRNs, renders our proposed approach suitable for allocating resources in dynamic social networks supported by wireless infrastructures. Furthermore, in this framework, social information may be leveraged to either improve the resource allocation algorithm itself or facilitating the social communication and information diffusion. Several works in the literature study this interplay between social information and cross-layer protocol design in wireless networks. This interplay is most representatively expressed when referring to mobile social networks, explored in [5] where it is proposed that the latter can further leverage spectrum access opportunities via CRNs enhanced with social information. Spectrum access in CRNs can be efficiently improved via channel recommendation mechanisms performed distributively and mimicking analogous schemes in electronic commerce as proposed in $[21,22]$. However, channel recommendations are meaningful when secondary users bear a limited view of spectrum opportunities (e.g., absence of a central database providing information for vacant channels and their conditions). Assuming a central database for channel information, [23] proposes a distributed channel access algorithm based on the fact that each user maximizes a utility function with respect to the experienced interference by itself and its social ties. Finally, the impact of social selfishness on the cross-layer design of CRNs has been studied in [24]. Social selfishness refers to the preference of users to relay data belonging only to their social friends and implies new constraints in the cross-layer problem regarding the allocated buffer sizes and the relay rates between user pairs. In [24], the derived algorithm is founded on a weighted BP scheme. Based on the latter algorithm but following the lines of a similar approach in [25], the weighted BP technique is applied for introducing social awareness in our proposed backpressure based resource allocation scheme for CRNs.

The main results and contributions of this paper can be summarized as follows:

- We propose the BPeMRF algorithm performing routing, scheduling, channel allocation, and power control via exploiting BP features and using an MRF decision model. BPeMRF is shown to be throughput optimal and is performed distributively while retrieving the minimum possible information from a centralized spectrum database.

- We improve the complexity of applying the BP algorithm, by alleviating the need for the NP-hard computation which is required to find the independent sets of links of the network graph demanded by the MWM. This is performed by replacing the 
MWM with the distributed Gibbs sampling method which provably converges and does not impact the throughput optimality of the BP algorithm.

- We enhance the BPeMRF algorithm with information from social layer, proposing the SeBPeMRF algorithm. The queue stability and its emerging trade-off with social behavior under SeBPeMRF are analyzed, while two case studies evaluate the two different objectives of incorporating the social information. The first objective is to improve the CRN operation via exploiting information of the social layer, while the second one is to exploit the agility of CRNs towards strengthening the efficiency of routing and information dissemination according to social layer information.

\section{System model}

Following the latest trends in dynamic spectrum environments, we adopt a CR databaseassisted system model conforming to recent trends for exploiting unlicensed TV whitespaces [11]. Whitespace databases gather spectrum sensing data, provide spectrum awareness, and adapt secondary user (SU) transmit profiles in order to ensure seamless primary user (PU) operation. Additionally, they can be potentially used to provide social-related and properly adapted information.

We consider $N$ SUs within an unlicensed opportunistic spectrum access model of $M$ heterogeneous channels, while each channel $m$ is characterized by its own central frequency carrier $f_{m}$ and bandwidth $W_{m}$. SUs coexist with higher priority PUs, potentially of different technologies, and communicate with a centralized network controller (white space database), as shown in Figure 1, which is responsible for providing them specific operational parameters, i.e., channel availability list and power restrictions per channel depending on primary activity and SUs location. We denote by $P_{i, m}$ the maximum permitted power at channel $m$ at which node $i$ can transmit without causing interference to PUs.

Apart from conforming to regulation limitations - hence, incorporating the underlying limitations posed by primary activity - we go one step ahead by aiming at optimizing the performance of the secondary network. In this direction, we suppose that each $\mathrm{SU} i$ is capable of selecting channel $m$ and performing power control under the constraints of $P_{i, m}$. We assume that the range of permitted power is discretized into $L$ levels, i.e., $a_{P}^{(i)} \in$ $\left\{a_{P_{1}}, \cdots, a_{P_{L}}\right\}$ where $a_{P}^{(i)}$ denotes a percentage value (e.g., $a_{P_{1}}=0 \%, a_{P_{L}}=100 \%$ ) of the

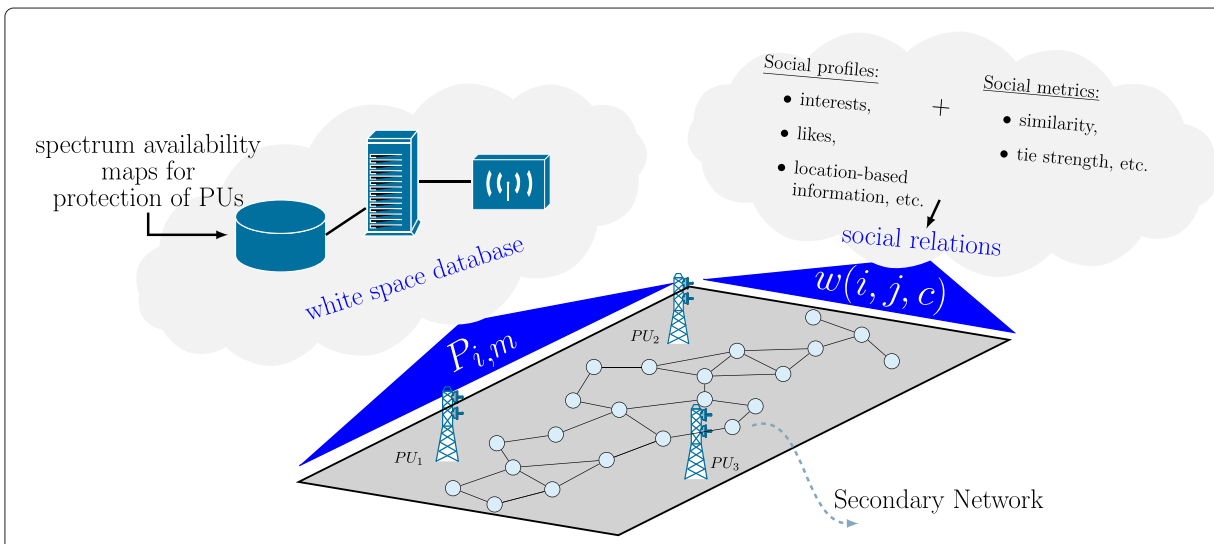

Figure 1 Considered system architecture. 
maximum permitted power $P_{i, m}$. Thus, using a simplified path loss model and denoting by $P_{R}^{t h r}$ the SU receiver sensitivity threshold, as determined by hardware specifications, the transmission range $R_{T_{i}}^{m}$ of node $i$ over channel $m$ can be estimated as:

$$
R_{T_{i}}^{m}=d_{0}\left[\frac{a_{P}^{(i)} P_{i, m} G_{t} G_{r}}{P_{R}^{t h r}} \cdot\left(\frac{c_{0}}{4 \pi d_{0} f_{m}}\right)^{2}\right]^{\frac{1}{\eta}}
$$

where $c_{0}$ represents the speed of light, $d_{0}$ the line-of-sight (LOS) reference distance, and $\eta$ the path loss exponent. The antenna gains of the transmitter and receiver are denoted by $G_{t}$ and $G_{r}$, respectively.

Packet collisions at a SU receiver can occur whenever two or more packets are received at the same time. However, we assume that overlapping transmissions in the secondary network are possible due to the capture effect. More precisely, in case of two concurrent SU transmissions, a SU can still receive a packet without collision if the SINR at the receiver is high enough for successfully decoding the ongoing transmission. Therefore, in our analysis, we adopt the capture threshold model [26], by which SU $i$ can successfully transmit to $j$ over channel $m$ if

$$
a_{P}^{(i)} \cdot P_{i, m} \cdot G_{i, j}^{(m)} \geq P_{R}^{t h r} \text { and } \frac{a_{P}^{(i)} \cdot P_{i, m} \cdot G_{i, j}^{(m)}}{a_{P}^{\left(i^{\prime}\right)} \cdot P_{i^{\prime}, m} \cdot G_{i^{\prime}, j}^{(m)}} \geq C_{p}^{t h r} \forall i^{\prime} \neq i
$$

where $C_{p}^{\text {thr }}$ denotes the capture threshold above which the interference from any potential transmitter $i^{\prime}$ can be ignored (not strong enough to create a collision). $G_{i, j}^{(m)}$ stands for the channel gain of link $(i, j)$ at channel $m$, i.e.,

$$
G_{i, j}^{(m)}=G_{t} \cdot G_{r} \cdot\left(\frac{c_{0}}{4 \pi d_{0} f_{m}}\right)^{2} \cdot\left(\frac{d_{0}}{d_{i j}}\right)^{\eta}
$$

where $d_{i j}$ is the Euclidean distance between SUs $i$ and $j$.

Regarding the SU traffic, we consider the existence of a finite number of commodities representing either flows, i.e., SU source-destination pairs, or destinations, within a time-slotted network operation. At each time slot $t$, new data are generated, and transmission decisions are made towards delivering all packets to their proper destinations. Specifically, the packets generated exogenously are assumed to arrive at each SU $i$ with rate $\lambda_{i}^{c}$, where $\lambda_{i}^{c} \neq 0$ only if $i$ is a source of packets for commodity $c$. Hence, the instantaneous number of packets arriving at source node $i$ at time $t$ for commodity $c$ is equal to $A_{i}^{c}(t)$, where $\mathbb{E}\left[A_{i}^{c}(t)\right]=\lambda_{i}^{c}$. The arrival rates are considered upper bounded, i.e., $\lambda_{i}^{c} \leq \lambda_{\max }, \forall i, c$. The set of sources for a commodity $c$ is denoted as $S(c)$ and $A_{i}^{c}(t)=0, \forall t, i \notin S(c)$. Furthermore, we denote the communication traffic in number of packets of every secondary link $s=(i, j)$ at time $t$ by $\mu_{i, j}(t)$ as upper bounded by $\mu_{i, j}(t) \leq \mu_{i, j}^{\max }=\max _{m}\left\{\left\lfloor\frac{W_{m}}{P k t_{s}} \cdot \log _{2}\left(1+\mathrm{SNR}_{s}\right)\right\rfloor\right\}$, where $P k t_{s}$ is the packet size and $\mathrm{SNR}_{s}=\frac{P_{i, m} \cdot G_{i, j}^{(m)}}{N_{0}+N_{P}} \cdot N_{0}$ denotes the background noise, while $N_{P}$ stands for the total additive interference/noise caused by the underlying primary system at each time slot $t$. Similarly, $\mu_{i, j}^{c}(t)$ stands for the communication traffic over secondary link $s=(i, j)$ regarding only commodity $c$. The packets for commodity $c$ that have arrived at SU $i$, but have not yet been forwarded, are stored in a commodity-specific queue at node $i$, denoted by $Q_{i}^{c}$, with length $Q_{i}^{c}(t)$ at time slot $t$. If $i$ is the destination of the commodity $c$, then $Q_{i}^{c}(t)=0, \forall t$. 
Furthermore, as shown in Figure 1, we assume the existence of social information overlaying the CRN. This social information is interpreted according to the considered application (e.g., sections 'Case study I: reputation of PUs appearance', 'Case study II: adapting routing efficiency to social layer's needs'), but it is always quantified via appropriate weight values. Specifically, a weight $w(i, j, c)$ is assigned to each pair of secondary link $s=(i, j)$ and commodity $c$ expressing a social relation. The definition of social relation regards the similarity among the social profiles of the SUs corresponding to the secondary link $s$ and the commodity $c$. The term social profile includes all the characteristics of the corresponding SU, i.e., its interests and likes (e.g., section 'Case study II: adapting routing efficiency to social layer's needs') or even its location-based contextual services such as acquired information about reputations and online recommendations (e.g., section 'Case study I: reputation of PUs appearance'). In our case studies, the social profiles can be stored locally at each SU. Note that, in this work, we are not concerned with the derivation of the social profiles which are considered given. Indicatively, the social profiles can be constructed using real data information, such as Facebook profiles. A detailed description of the properties of the weights' values is provided in the 'Exploiting social information in CRN cross-layer optimization and resource allocation' section.

\section{MRF-based resource allocation framework for centralized CRNs}

In this section, we delve into the analysis of the secondary network operation through an MRF formulation which can properly incorporate the inherent spatial dependencies of the examined system model. To improve the secondary network efficacy, the proposed cross-layer BPeMRF framework is introduced by explaining its components and analyzing its main properties.

\section{MRF formulation for secondary networks}

Constrained by the underlying limitations posed by primary activity, SUs make adaptations and continuously take decisions which unavoidably influence neighboring SUs. In this manner, a knock-on effect is generated by which local decisions give rise to long-range adaptations and essentially can contribute to the overall secondary network performance. This behavior unveils spatial dependencies on SU operation along with a generalized Markov property that motivate our following MRF-centered analysis and formulation.

Based on the maximum transmission range of each $\mathrm{SU} i, R_{T_{i}}^{(\max )}$, which can be estimated by Equation (1), we define by $\mathcal{K}$ the set of all possible directed secondary links, with cardinality $K$. Each link $s=(i, j) \in \mathcal{K}$ is mapped to an MRF site $s$ and represents a communication link with transmitter node $i$ and receiver node $j$. MRFs describe a probabilistic measure in a family of spatially dependent random variables $X_{s}$ which are associated with a finite number of MRF sites $s \in S$, where $S \doteq \mathcal{K}$ in our case. Every random variable $X_{s}$ takes values $x_{s}$, also referred to as states, from a finite space $\Lambda$, whereas the combination of states of all MRF sites describes a configuration $\omega=\left(x_{1}, \ldots, x_{s}, \ldots, x_{n}\right) \in$ $\Omega=\left\{\left(x_{1}, \ldots, x_{s}, \ldots, x_{n}\right): x_{s} \in \Lambda, s \in S\right\}$ that corresponds to one of all possible states of the whole system. In the same manner for our formulation, the state of a secondary link $s=(i, j)$ is expressed by the 2-tuple $\left\langle m, a_{P}\right\rangle_{s}$ with state space size equal to $M \times L$. It represents the selected channel $m$ and the real power (after power control) of the transmitter $i$ as the percentage of the maximum permitted power $P_{i, m}$. Thus, for example, if site $s=(i, j)$ has state $\langle 1,50 \%\rangle$, it means that the corresponding link is active and node $i$ 
transmits to node $j$ at channel 1 (namely, at frequency carrier $f_{1}$ and channel bandwidth $W_{1}$ ) with transmission power equal to $0.5 \times P_{i, 1}$.

The main property of MRFs is that the state of each site depends only on a local set of neighbors and is expressed by the following conditional probabilities (also called local characteristics),

$$
\mathbb{P}\left(X_{s}=x_{s} \mid X_{r}=x_{r}, r \neq s\right)=\mathbb{P}\left(X_{s}=x_{s} \mid X_{r}=x_{r}, r \in \mathcal{G}_{s}\right)
$$

where $\mathcal{G}_{s}$ stands for the MRF neighborhood of each site $s$ and satisfies the conditions: $\forall s \in S, s \notin \mathcal{G}_{s}$ and $r \in \mathcal{G}_{s}$ if and only if $s \in \mathcal{G}_{r}$. In our case, we define a neighborhood system $\mathcal{G}=\left\{\mathcal{G}_{s}\right\}_{s \in \mathcal{K}}$ such that two sites (secondary links) $s$ and $s^{\prime}$ are MRF neighbors if and only if their concurrent operation is possible to affect the network performance in terms of packet collisions. Thus, the MRF neighborhood of $s$ is defined as:

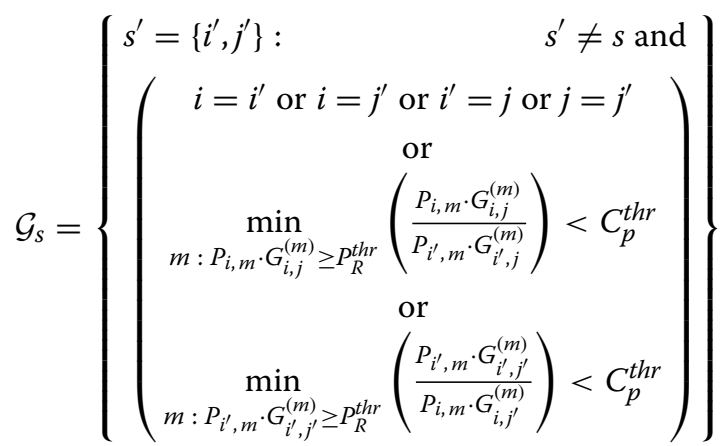

Any random field with property (4) on a neighborhood system $\mathcal{G}$ can be also represented as a Gibbs field of the form

$$
\mathbb{P}(X=\omega)=\frac{1}{Z} e^{-\frac{U(\omega)}{T}}
$$

with a suitable choice of energy function $U(\omega)$ that can be further decomposed into the contributions of smaller subsets of $S, V_{C}(\omega)$, also called potential functions. $Z:=$ $\sum_{\omega \in \Omega} e^{-\frac{U(\omega)}{T}}$ denotes the partition function and $T$ a system parameter, also referred to as temperature. In this work, we leverage the class of pairwise, nearest-neighbor potentials and decompose the system energy into neighbor pair potentials, i.e., $V_{C}=0$ if $C$ is not a clique or $|C|>2$, as follows:

$$
U(\omega)=\sum_{s \in S} V_{\{s\}}^{(1)}\left(x_{s}\right)+\sum_{\left\{s, s^{\prime}\right\} \in(S \times S), s^{\prime} \in \mathcal{G}_{s}} V_{\left\{s, s^{\prime}\right\}}^{(2)}\left(x_{s}, x_{s^{\prime}}\right)
$$

Aiming at uniquely specifying our MRF formulation, we design the corresponding singleton $V_{\{s\}}^{(1)}$ and doubleton potentials $V_{\left\{s, s^{\prime}\right\}}^{(2)}$ towards capturing via the energy function the different contributions of system configurations $\omega$ in the secondary network capacity, as well as the cost of potential packet collisions. Thus, the singleton potential for each link $s=(i, j)$ with state $x_{s}=\left\langle m, a_{P}\right\rangle_{s}=\left\{m, a_{P}^{(s)}\right\}$ is expressed by

$$
V_{\{s\}}^{(1)}\left(x_{s}\right)=\left\{\begin{array}{lc}
\delta_{1}>0, & \text { if } 0<a_{P}^{(s)} \cdot P_{i, m} \cdot G_{i, j}^{(m)}<P_{R}^{t h r} \\
-\lambda_{1} \cdot \frac{W_{m}}{C_{\max }} \cdot \log _{2}\left(1+\frac{a_{P}^{(s)} \cdot P_{i, m} \cdot G_{i, j}^{(m)}}{N_{0}+N_{P}}\right), & \text { otherwise. }
\end{array}\right.
$$

In the above formulation, each state of an MRF site (secondary link) contributes to the system energy according to the maximum possible link capacity that could offer, whereas 
$\delta_{1}$ represents a penalty for activating useless links, i.e., incapable of successfully delivering packets. $C_{\max }$ denotes the maximum link capacity in the secondary network and serves for normalization purposes. Similarly, the doubleton potential $V_{\left\{s, s^{\prime}\right\}}^{(2)}$ is designed to capture the interaction between secondary communication links, e.g., link $s$ with state $x_{s}=\left\langle m, a_{P}\right\rangle_{s}=\left\{m, a_{P}^{(s)}\right\}$ and link $s^{\prime}$ with $x_{s^{\prime}}=\left\langle m, a_{P}\right\rangle_{s^{\prime}}=\left\{m^{\prime}, a_{P}^{\left(s^{\prime}\right)}\right\}$, as follows

$$
V_{\left\{s, s^{\prime}\right\}}^{(2)}\left(x_{s}, x_{s^{\prime}}\right)= \begin{cases}\lambda_{2} \cdot \delta_{2}>0, & \text { if 'collisionCondition' }=1 \\ 0, & \text { otherwise. }\end{cases}
$$

$\delta_{2}$ is a large positive constant value that penalizes collisions between two active secondary links by increasing the system energy. The 'collisionCondition' describes the possible scenarios/configurations between $s$ and $s^{\prime}$ that can lead to packet collision and is expressed by

$$
\begin{array}{r}
\left(\begin{array}{c}
a_{P}^{(s)} \neq 0 \text { and } a_{P}^{\left(s^{\prime}\right)} \neq 0 \text { and } \\
\left(i=i^{\prime} \text { or } i=j^{\prime} \text { or } i^{\prime}=j \text { or } j=j^{\prime}\right)
\end{array}\right) \text { OR } \\
m=m^{\prime} \text { and } \\
\left(\begin{array}{c}
P_{R}^{t h r} \leq\left[a_{P}^{(s)} \cdot P_{i, m} \cdot G_{i, j}^{(m)}\right]<C_{p}^{t h r} \cdot\left[a_{P}^{\left(s^{\prime}\right)} \cdot P_{i^{\prime}, m} \cdot G_{i^{\prime}, j}^{(m)}\right] \\
\text { or } \\
P_{R}^{t h r} \leq\left[a_{P}^{\left(s^{\prime}\right)} \cdot P_{i^{\prime}, m} \cdot G_{i^{\prime}, j^{\prime}}^{(m)}\right]<C_{p}^{t h r} \cdot\left[a_{P}^{(s)} \cdot P_{i, m} \cdot G_{i, j^{\prime}}^{(m)}\right]
\end{array}\right)
\end{array}
$$

It is noted that $\lambda_{1}$ and $\lambda_{2}$ represent non-negative parameters to control the strength of potentials' contributions.

The above MRF formulation of the secondary network facilitates the estimation of optimal solutions exploiting powerful MRF energy minimization techniques in an otherwise difficult to be solved problem. Given that the space of possible configurations can be very large, the MRF formulation can leverage algorithms for the computation of global optimum solutions relying on repeated computation of local characteristics (Equation 4). As a result, by finding configurations (i.e., specifying channel allocations and power control at each secondary link) with minimum MRF system energy, we are able to both maximize the offered capacity per link and provide to SUs an efficient scheduling scheme avoiding collisions and, hence, improve the overall secondary network performance.

In this work, we investigate optimal configurations with minimum MRF energy based on a stochastic relaxation methodology, namely Gibbs sampling [9]. The key idea behind Gibbs sampling is that following a visiting scheme, each currently visited MRF site, $s$, updates its own state according to the local conditional probability distribution

$$
\mathbb{P}\left(X_{s}=x_{s} \mid X_{r}=x_{r}, r \neq s\right)=\frac{1}{Z_{s}} \cdot \exp \left(-\frac{1}{T(\text { sweepID })} \cdot \sum_{C: s \in C} V_{C}\left(\omega^{x_{s}}\right)\right)
$$

where $Z_{s}=\sum_{x_{s} \in \Lambda} \exp \left(-\frac{1}{T \text { (sweepID) }} \cdot \sum_{C: s \in C} V_{C}\left(\omega^{x_{s}}\right)\right)$. T(sweepID) represents an annealing schedule with decreasing rate of temperature $T$ for each sweep (indexed by sweepID) that denotes the time interval within which all sites have updated their states. $\omega^{x_{s}}$ stands for the configuration which has value $x_{s}$ at site $s$ and agrees with $\omega$ everywhere else. It has been theoretically proven in [9] that by applying Gibbs sampling with a suitable logarithmic annealing, the system converges to minimum energy configurations. 


\section{Potential function via back pressure}

In this section, we delve into the design of the potential functions (section 'MRF formulation for secondary networks') targeting at introducing throughput optimality in the proposed scheduling scheme (via power control) of the 'MRF formulation for secondary networks' section. Towards this direction, we adapt the main idea of the throughput optimal back pressure algorithm [10] which performs routing and link scheduling based on the congestion gradients (queue backlog differentials) computed on every communication link. Specifically, BP chooses for transmission at each time slot, a maximal independent set of links (non-interfering links) that achieves the maximum sum of queue backlog differentials multiplied with the corresponding link's communication traffic. At each time slot $t$, after the computation of the communication traffic variables, $\mu_{i, j}^{c}(t), \forall s=(i, j), c$ (section 'System model'), according to the chosen schedule, each $\mathrm{SU} i$ renews its queue for commodity $c$ following the renewal relation:

$$
Q_{i}^{c}(t+1) \leq \max \left\{Q_{i}^{c}(t)-\sum_{j} \mu_{i, j}^{c}(t), 0\right\}+\sum_{j} \mu_{j, i}^{c}(t)+A_{i}^{c}(t) .
$$

Throughput optimality is tied with the strong stability of queues. A queue, $Q_{i}^{c}$, is strongly stable if lim $\sup _{t->\infty} \frac{1}{t} \sum_{\tau=0}^{t-1} E\left(Q_{i}^{c}(\tau)\right)<\infty$ [27]. If all the queues of the network are strongly stable, then the whole network is strongly stable. The capacity region, $\mathcal{C}$, of the network is defined as the set of source rates $\left\{\lambda_{i}^{c}, \forall i, c\right\}$, for which there exists a control algorithm that can stabilize the network [20]. Any algorithm that can support every source rate inside $\mathcal{C}$ while maintaining stability is called throughput optimal [20].

Aiming to leverage the throughput optimality of the BP algorithmic design, we modify the singleton and doubleton potential functions in Equations 8 and 9 by replacing the constant homogeneous parameters $\lambda_{1}, \lambda_{2}$, with the heterogeneous, time-varying queue backlogs defined on each corresponding site of the MRF graph. In this case, the following proposition holds.

Proposition 1. Define the parameter $\lambda_{1}$ in the MRF singleton potentials (Equation 8) separately for each site $s=(i, j)$ and the parameter $\lambda_{2}$ in the MRF doubleton potentials (Equation 9) separately for each pair of sites $s=(i, j), s^{\prime}=\left(i^{\prime}, j^{\prime}\right)$, as $\lambda_{1}=$ $\max \left\{\max _{c}\left\{Q_{i}^{(c)}-Q_{j}^{(c)}\right\}, 0\right\}$ and $\lambda_{2}=\max \left\{\max _{c}\left\{Q_{i}^{(c)}-Q_{j}^{(c)}\right\}, \max _{c}\left\{Q_{i^{\prime}}^{(c)}-Q_{j^{\prime}}^{(c)}\right\}, 1\right\}$, while additionally $V_{\{s\}}^{(1)}\left(x_{s}\right)=\delta_{1} ; \forall x_{s}$ if $\max _{c}\left\{Q_{i}^{(c)}-Q_{j}^{(c)}\right\}<0$. Then, the proposed BPeMRF is throughput optimal, i.e., it stabilizes the queues, assuming that the arrival rates on the SUs lie inside the capacity region $\mathcal{C}$.

Proof. The MRF energy minimization problem becomes

$$
\min _{\omega \in \Omega} \sum_{s \in S} V_{\{s\}}^{(1)}\left(x_{s}\right)+\sum_{s \in S} \sum_{s^{\prime} \in \mathcal{G}_{s}} V_{\left\{s, s^{\prime}\right\}}^{(2)}\left(x_{s}, x_{s^{\prime}}\right)
$$

Since $\delta_{1}$ and $\delta_{2}$ are large positive values, the corresponding configurations that activate such penalties cannot be part of the minimum energy solution. Note that $\lambda_{2}$ is tuned by Proposition 1 such that it is always in the order of $\lambda_{1}$ to avoid unbalance between the contributions of singleton and doubleton potentials. Let us denote with $\Gamma$ the set of configurations adding cost equal to $\delta_{1}$ or $\delta_{2}$ in the total MRF energy based on Equations 
8 and 9 . Then, the search space of the optimal solution can be restricted by rewriting our initial minimization problem as

$$
\begin{aligned}
& \min _{\omega \in \Omega} \sum_{\{i, j\} \in S}\left[-\lambda_{1} \cdot W_{m} \cdot \log _{2}\left(1+\frac{a_{P}^{(s)} \cdot P_{i, m} \cdot G_{i, j}^{(m)}}{N_{0}+N_{P}}\right)\right] \\
& \text { subject to } \omega \notin \Gamma
\end{aligned}
$$

Since the constraints expressed by $\Gamma$ guarantee interference avoidance between two active secondary links (based on capture threshold model), the experienced SNR of an active link $s$ is equal to $\mathrm{SNR}_{s}=\frac{a_{P}^{(s)} \cdot P_{i, m} \cdot G_{i, j}^{(m)}}{N_{0}+N_{P}}$. Since $\mu_{i, j}(t)=\frac{W_{m}}{P k t_{s}} \cdot \log _{2}\left(1+\mathrm{SNR}_{s}\right)$ is the communication traffic over site $s=(i, j)$ at $t$ given the chosen channel $m$ and the power level $a_{P}^{(s)}$ (section 'System model') and by defining that $\mu_{i, j}(t)=0$ for all $\{i, j\}$ node pairs with distance greater than their maximum communication range (i.e., $d_{i, j}>R_{T_{i}}^{(\max )}$ ), the minimization problem is rewritten as

$$
\min _{\omega \in \Omega} \sum_{i} \sum_{j}\left[-\lambda_{1} \cdot \mu_{i, j}(t)\right], \quad \text { subject to } \omega \notin \Gamma
$$

Following the assumption of the proposition, by tuning the parameter $\lambda_{1}$ of each singleton as $\lambda_{1}=\max \left\{\max _{c}\left\{Q_{i}^{(c)}-Q_{j}^{(c)}\right\}, 0\right\}$ for each site $s=(i, j)$, the MRF minimization is transformed to the following maximization:

$$
\begin{aligned}
& \max _{\omega \in \Omega} \sum_{i} \sum_{j}\left[\mu_{i, j}(t) \cdot \max \left\{\max _{c}\left\{Q_{i}^{(c)}(t)-Q_{j}^{(c)}(t)\right\}, 0\right\}\right] \\
& \text { subject to } \omega \notin \Gamma
\end{aligned}
$$

The final equivalent maximization problem is identical to the back pressure routing/scheduling policy which is throughput optimal [10], thus concluding the proof.

It is important to mention that the proposed design of the potential functions allows for the routing component to be included in the BPeMRF cross-layer scheme, in an exactly similar way as in the BP algorithm, while also leading to throughput optimality regarding scheduling. More specifically, routing is introduced via the choice of the optimal commodity to be served by each site. Each link (site) $s=(i, j)$ computes its queue back$\log$ differential for all commodities, $R_{i j}^{c}(t)=Q_{i}^{c}(t)-Q_{j}^{c}(t), \forall c$ and the maximum one among all commodities $R_{i j}(t)=\max \left\{\max _{c} R_{i j}^{c}(t), 0\right\}$ is inserted in the parameters $\lambda_{1}, \lambda_{2}$ (Proposition 1) for the decisions regarding power and channel assignments to be made (scheduling is enclosed in the power control) via the MRF energy minimization. Finally, the commodity $c^{*}(i, j)=\arg \max _{c} R_{i j}^{c}(t)$ will be chosen for service if the site $s=(i, j)$ is scheduled to transmit (i.e., the optimization concludes to non-zero power assignment to node $i$. Therefore, $\mu_{i, j}^{c}(t)=\mu_{i, j}(t)$ if $c=c^{*}(i, j)$, else $\mu_{i, j}^{c}(t)=0$.

On the other hand, our contribution is favorable for the back pressure algorithm itself and its practical implementation. This is due to the fact that the optimal realization of $\mathrm{BP}$ requires the solution of a MWM, the centralized implementation of which is NPhard [19]. BPeMRF can be alternatively seen as the replacement of the MWM in the $\mathrm{BP}$ algorithm with the less complex Gibbs sampling technique which can be performed 
distributively on each node based on some globally provided information (spectrum database, section 'System model'). This fact constitutes an important contribution of the BPeMRF scheme in the fields of CRNs and dynamic social networks where the dynamic network topology requires the repetitive NP-hard computation of the maximal independent sets of the underlying physical layer graph for the solution of the MWM. However, since the Gibbs sampler needs theoretically an infinite number of sweeps to converge, its finite application will provide an approximation of the optimal MWM solution.

Based on the previous observations, in the 'Simulation results' section, we compare the performance of the BPeMRF scheme (approximation of the MWM) with the back pressure routing and scheduling algorithm (optimal computation of the MWM). However, contrary to the BPeMRF scheme, BP in its canonical form does not perform channel selection. To tackle this issue and only for comparison reasons, we proceed with the redesign of the BP algorithm as follows.

Let us define a binary function $I_{s}$, where $I_{s}(m, t)=1$ if the site $s=(i, j)$ uses the channel $m$ at time slot $t$, else $I_{s}(m, t)=0$. Also, $\sum_{m} I_{s}(m, t)=1$ (section 'System model'). Let $\mu_{i, j}^{m}(t)$ be the maximum communication traffic of site $s=(i, j)$ over channel $m$ at time $t$. Then, scheduling is decided by solving the MWM: $\max _{I} \sum_{(i, j)}$ $\left\{\sum_{m} \mu_{i, j}^{m}(t) I_{i, j}(m, t)\right\} R_{i j}(t)$, subject to interference constraints and $\sum_{m} I_{s}(m, t)=1$, $I_{S}(m, t) \in\{0,1\}, \forall s$. In order to solve the MWM, we consider the graph of SUs where each link $(i, j)$ is replaced by $M$ links, each one corresponding to a channel $m \in\{1 \ldots M\}$, and denoted by $(i, j, m)$. Then, we appropriately define the maximal independent sets (set $I D(t))$ over the interference constraints of the new graph, i.e., each site can use only one channel and half-duplex communication, and the constraints of the capture model (section 'System model') for the links transmitting at the same channel. Let us denote with $\mu_{i, j, m}(t)=\mu_{i, j}^{m}(t)$ the communication traffic of link $(i, j, m)$ and $R_{i j m}(t)=R_{i j}(t), \forall m$. Then according to the above, the initial MWM is equivalent to the MWM defined as $\max _{\mu \in \mathbf{I D}(\mathbf{t})} \sum_{(i, j, m)} \mu_{i, j, m} R_{i j m}(t)$, and therefore the latter needs to be solved. Each site $s=(i, j)$ for which $\mu_{i, j, m}(t) \neq 0$ for a channel $m$, serves over channel $m$, the commodity $c^{*}(i, j)$ with rate $\mu_{i, j}^{m}(t)$.

\section{Exploiting social information in CRN cross-layer optimization and resource allocation}

In this section, BPeMRF is enhanced via leveraging users' information from the social layer targeting at a cross-layer improvement of our proposed framework. In order to achieve the social awareness of BPeMRF, the parameters $\lambda_{1}$ and $\lambda_{2}$ will be redesigned to incorporate the weight values described in the 'System model' section (Figure 1), which are able to express diverse social relationships based on their definition. Specifically, the queue differential corresponding to site $s=(i, j)$ for the commodity $c$ is scaled by the weight $w(i, j, c)$, i.e.,

$$
\lambda_{1}^{a}=\max \left\{\max _{c}\left\{w(i, j, c)\left(Q_{i}^{(c)}-Q_{j}^{(c)}\right)\right\}, 0\right\}
$$

and similarly, $\lambda_{2}^{a}$ depending on sites $\left\{s, s^{\prime}\right\}$ is expressed by

$$
\lambda_{2}^{a}=\max \left\{\max _{c}\left\{w(i, j, c)\left(Q_{i}^{(c)}-Q_{j}^{(c)}\right)\right\}, \max _{c}\left\{w\left(i^{\prime}, j^{\prime}, c\right)\left(Q_{i^{\prime}}^{(c)}-Q_{j^{\prime}}^{(c)}\right)\right\}, 1\right\}
$$


The BPeMRF algorithm where $\lambda_{1}$ and $\lambda_{2}$ are replaced by $\lambda_{1}^{a}$ and $\lambda_{2}^{a}$ correspondingly forms the SeBPeMRF algorithm. This approach aims at prioritizing in routing and scheduling commodities and links presenting social relationships of higher quality, i.e., higher values of $w(i, j, c), \forall i, j, c$, while also considering the congestion indices as expressed by the queue differentials. As will be shown via simulation results, it can effectively improve network performance by intensely expressing the heterogeneity of secondary links with respect to their contribution in performance metrics (e.g., routing through socially connecting nodes, delay, throughput, quality of routing, etc.). However, it is proven that network stability in a weighted BP technique is achieved only for a part of the capacity region [25].

Regarding the weights $w(i, j, c)$, the following assumptions should be satisfied. Firstly, their values are assumed invariant with time. Secondly, for each commodity $c$, there will be values $w_{\min }^{c}, w_{\max }^{c}$ such that $w_{\min }^{c} \leq w(i, j, c) \leq w_{\max }^{c}, \forall i, j$. By defining $\rho^{c}=\frac{w_{\max }^{c}}{w_{\min }^{c}}, \forall c$, it is observed that $\rho^{c}$ is a metric of the heterogeneity of the weight values across all network links for the commodity $c$. Specifically, if $\rho^{c}$ has a high value, then the commodity $c$ maintains more heterogeneous social relations in the network compared with a small $\rho^{c}$ value. In the special case where the weights do not depend on the commodities, i.e., $w(i, j, c)=w(i, j), \forall c$, then $\rho^{c}=\frac{w_{\max }}{w_{\min }}, \forall c$, where $w_{\min }=\min _{\forall i, j} w(i, j), w_{\max }=$ $\max _{\forall i, j} w(i, j)$.

Proposition 2 determines the sufficient condition for queue stability to hold under SeBPeMRF.

Proposition 2. Assume $\varepsilon^{c}>0, \forall c$ such that $\frac{\varepsilon^{c}}{\lambda_{i}^{c}} \geq\left(\rho^{c}-1\right), \forall c, i \in S(c)$. If the set $\left\{\lambda_{i}^{c}+\varepsilon^{c}\right\}_{\forall c, i \in S(c)}$ lies inside the capacity region $\mathcal{C}$, then strong queue stability holds for the proposed policy.

The proof of Proposition 2 follows similar lines with the proof of Theorem 1 in [25], and therefore, it is briefly described.

Proof. Since by Proposition 1, we have shown that BPeMRF behaves as BP, it suffices to prove the statement of Proposition 2 for the BP algorithm.

We define the Lyapunov function $L(Q(t))=\sum_{i, c} w_{\max }^{c} Q_{i}^{c}(t)^{2}$ and we compute the Lyapunov drift

$$
\begin{aligned}
E(L(Q(t+1))-L(Q(t)) \mid Q(t))= & E\left[\sum_{i, c} w_{\max }^{c}\left(Q_{i}^{c}(t+1)^{2}-Q_{i}^{c}(t)^{2}\right) \mid Q(t)\right] \\
\leq & B_{\max }+2 \sum_{i, c} w_{\max }^{c} Q_{i}^{c}(t) \lambda_{i}^{c}-2 \sum_{i, c} w_{\max }^{c} Q_{i}^{c}(t) \\
& \times E\left[\left(\sum_{j} \mu_{i j}^{c}(t)-\sum_{j} \mu_{j i}^{c}(t)\right) \mid Q(t)\right] \\
= & B_{\max }+2 \sum_{i, c} w_{\max }^{c} Q_{i}^{c}(t) \lambda_{i}^{c}-2 \sum_{c} \sum_{(i, j)} w_{\max }^{c} \\
& \times E\left[\mu_{i j}^{c}(t) \mid Q(t)\right]\left(Q_{i}^{c}(t)-Q_{j}^{c}(t)\right),
\end{aligned}
$$

where $B_{\max }=\max \left\{\sum_{i, c} w_{\text {max }}^{c} E\left[\left(\sum_{j} \mu_{i j}^{c}(t)\right)^{2}+\left(\sum_{j} \mu_{j i}^{c}(t)+A_{i}^{c}(t)\right)^{2} \mid Q(t)\right]\right\}$. 
For the communication traffic variables $\mu_{i j}^{c}(t), \forall i, j, c$ chosen by SeBPeMRF, it holds that

$$
\sum_{c} \sum_{(i, j)} w(i, j, c) \mu_{i j}^{c}(t)\left(Q_{i}^{c}(t)-Q_{j}^{c}(t)\right) \leq \sum_{c} \sum_{(i, j)} w_{\max }^{c} \mu_{i j}^{c}(t)\left(Q_{i}^{c}(t)-Q_{j}^{c}(t)\right),
$$

since for the SeBPeMRF $\mu_{i j}^{c}(t)=0$ if $Q_{i}^{c}(t)-Q_{j}^{c}(t)<0$ and therefore from Rel. (14) it holds that

$$
\begin{array}{r}
E(L(Q(t+1))-L(Q(t)) \mid Q(t)) \leq \\
B_{\max }+2 \sum_{i, c} w_{\max }^{c} Q_{i}^{c}(t) \lambda_{i}^{c}-2 \sum_{c} \sum_{(i, j)} w(i, j, c) \mu_{i j}^{c}(t)\left(Q_{i}^{c}(t)-Q_{j}^{c}(t)\right) .
\end{array}
$$

If $\lambda_{i}^{c}+\epsilon^{c}, \forall c, i \in S(c)$ lie inside the capacity region, then from Corollary 3.9 in [27], there exist communication traffic variables $\hat{\mu}_{i j}^{c}(t)$ (according to a stationary randomized control policy making decisions on the current network state) which satisfy:

$$
\lambda_{i}^{c}+\epsilon^{c}=E\left[\sum_{j} \hat{\mu}_{i j}^{c}(t)-\sum_{j} \hat{\mu}_{j i}^{c}(t)\right] \forall c, i \in S(c) .
$$

As a result, we have

$$
\begin{aligned}
\sum_{i, c} w_{\min }^{c} Q_{i}^{c}(t) \lambda_{i}^{c}+\sum_{i, c} w_{\min }^{c} Q_{i}^{c}(t) \epsilon^{c} \\
\quad=\sum_{c} \sum_{(i, j)} w_{\min }^{c} E\left[\hat{\mu}_{i j}^{c}(t)\right]\left(Q_{i}^{c}(t)-Q_{j}^{c}(t)\right) \\
\leq \max _{\mu^{\prime}} \sum_{c} \sum_{(i, j)} w_{\min }^{c} \mu_{i j}^{c \prime}\left(Q_{i}^{c}(t)-Q_{j}^{c}(t)\right) \\
\leq \max _{\mu^{\prime}} \sum_{c} \sum_{(i, j)} w(i, j, c) \mu_{i j}^{c \prime}\left(Q_{i}^{c}(t)-Q_{j}^{c}(t)\right) \\
=\sum_{c} \sum_{(i, j)} w(i, j, c) \mu_{i j}^{c}(t)\left(Q_{i}^{c}(t)-Q_{j}^{c}(t)\right),
\end{aligned}
$$

where $\mu_{i j}^{c}(t), \forall i, j, c$ are chosen by SeBPeMRF. Now, the Lyapunov drift (Rel. (16)) becomes

$$
\begin{aligned}
E[L(Q(t+1))-L(Q(t)) \mid Q(t)] \leq & B_{\max }+2 \sum_{i, c}\left(w_{\max }^{c}-w_{\min }^{c}\right) \lambda_{i}^{c} Q_{i}^{c}(t) \\
& -2 \sum_{i, c} w_{\min }^{c} Q_{i}^{c}(t) \epsilon^{c} .
\end{aligned}
$$

Therefore, if the assumption $\epsilon^{c}>\frac{w_{\max }^{c}-w_{\min }^{c}}{w_{\min }^{c}} \lambda_{i}^{c} \Rightarrow \frac{\epsilon^{c}}{\lambda_{i}^{c}}>\rho^{c}-1, \forall c, i \in S(c)$ is satisfied, then from Lemma 4.1 of [27], the network is strongly stable.

The two following remarks stem from Proposition 2.

Remark 1. 'Trade-off between social relations and queue stability'

Proposition 2 states that queue stability holds provably only for a part of the capacity region i.e., for $\varepsilon^{c}$ satisfying $\frac{\varepsilon^{c}}{\lambda_{i}^{c}} \geq\left(\rho^{c}-1\right), \forall c, i \in S(c)$ and not for $\varepsilon^{c}>0, \forall c$ which is a necessary and sufficient condition for throughput optimality [27]. Via Proposition 2, a trade-off emerges between the social behavior/relations and queue stability. Specifically, when $\rho^{c}$ increases for a commodity $c$, then $\frac{\varepsilon^{c}}{\lambda_{i}^{c}}, \forall i \in S(c)$ should also increase, implying 
lower possible exogenous arrival rates for commodity $c$. In other words, when the sources and/or the destination of a commodity c maintain more heterogeneous in quality social relations with the rest of the nodes (i.e., $\rho^{c}$ has high value for commodity $c$ ), there is less available long-term throughput (i.e., exogenous arrival rate) for this particular commodity so that the queues over the network are stabilized, given the maximum service rates of the network links. Thus, a commodity c corresponding to more homogeneous social relationships overall in the network, thus facing with similar weight values over the sites (or $\rho^{c} \rightarrow 1$ ), may exploit its best available long-term throughput which also depends on the rest of the sources' rates.

Remark 2. 'Closer examination of the effect of the weights' values'

If $w_{\max }^{c}$ increases for a commodity $c$, then this actually means that the set of sites corresponding to $w_{\max }^{c}$ will be more likely to be scheduled. This fact may lead to performance improvements for commodity c (i.e., throughput, delay, quality of routing etc.) depending also on the location and the number of sites belonging in this set. In general, for constant $\rho^{c}$, increasing the values of $w_{\max }^{c}$ and $w_{\min }^{c}$ is expected to affect positively the performance metrics regarding commodity c. On the contrary, for constant $w_{\max }^{c}$ and decreasing $w_{\text {min' }}^{c}$, the expected effect on the performance related to commodity $c$ is negative depending also on the number and the location of the sites assigned the changed values. This remark is examined via simulations in the case study II that follows. Finally, note that Proposition 2 reflects a worst-case scenario by using the minimum and maximum values of the weights and not their exact values so as to determine sufficient conditions that ensure queue stability. More explicitly, changing the weight values of a small number of links to very high or very low ones may not have the degree of impact as predicted by Proposition 2.

\section{Simulation results}

In this section, we evaluate the performance of the proposed BPeMRF and SeBPeMRF approaches through simulations on MATLAB over different networking scenarios. After studying the operation of our proposed framework in a trivial CRN topology to validate its applicability, we compare the network stability properties of BPeMRF with the traditional back pressure methodology (namely, based on MWM solution). In addition, two different case studies are examined in the 'Case study I: reputation of PUs appearance' and 'Case study II: adapting routing efficiency to social layer's needs' sections. The obtained results provide relevant operational performance indications for the proposed BPeMRF/SeBPeMRF approaches and demonstrate the significance/benefits of incorporating social information in CR infrastructures via cross-layer techniques.

For the rest of the experiments, we adopt a logarithmic annealing schedule for Gibbs sampling (similar in spirit to the theoretical annealing schedule [9]) and proceed until convergence or a predefined finite number of sweeps (e.g., 1,000 sweeps). Table 1 summarizes the setup of general system parameters (which are defined in the 'System model' and 'MRF-based resource allocation framework for centralized CRNs' sections) as used in the following simulations. In the examined networking scenarios, we assume $M=2$ homogeneous licensed channels with central frequency carriers $f_{1}=300 \mathrm{MHz}, f_{2}=320 \mathrm{MHz}$ and bandwidth equal to $50 \mathrm{kHz}$. It is noted that $P_{i, m}$ values are determined for each SU $i$ and channel $m$ based on the current PUs locations and transmission powers so as to always guarantee primary network seamless operation. In particular, we assume that PUs 
Table 1 Simulation parameters setup

\begin{tabular}{|c|c|c|}
\hline Parameters & Values & Definition \\
\hline$C_{p}^{\text {thr }}$ & 2 & Capture threshold - Equation 2 \\
\hline$P_{R}^{\text {thr }}$ & $-90 d B m$ & SU receiver sensitivity threshold - Equations 1 and 2 \\
\hline$N_{0}$ & $-98 d B m$ & Background noise - Equation 8 \\
\hline$G_{t}, G_{r}$ & 1 & Antenna gains - Equations 1 and 3 \\
\hline$d_{0}$ & $1 \mathrm{~m}$ & LOS reference distance - Equations 1 and 3 \\
\hline$\eta$ & 4 & Path-loss exponent - Equations 1 and 3 \\
\hline$\delta_{1}$ & 5 & Positive constant (penalty) - Equation 8 \\
\hline$\delta_{2}$ & 2 & Positive constant (penalty) - Equation 9 \\
\hline$P k t_{s}$ & 1,500 bytes & Packet size \\
\hline $\max \left\{P_{i, m}\right\}$ & $100 \mathrm{~mW}$ & maximum SU transmission power \\
\hline$T$ & $\frac{2}{\ln (1+\text { sweeplD })}$ & Annealing schedule - Equation 10 \\
\hline
\end{tabular}

transmit with power equal to $100 \mathrm{~mW}$, while the PU receiver sensitivity threshold is equal to $-74.2 \mathrm{dBm}$. To protect even the PUs receivers located at the boundary of PUs communication range (worst-case scenario), we set $P_{i, m}$ values such that SU's signal at each location is always at least $10 \mathrm{~dB}$ lower than the incumbent primary signal.

\section{Proof of concept in CRN environment}

To demonstrate the MRF energy minimization procedure and the underlying convergence behavior that results in global optimal secondary network configurations, we examine the trivial case of a line secondary topology (as shown in Figure 2), where the distance between neighboring SUs allows communication only at their maximum transmission power and thus, optimal configurations can be easily deduced. In the examined scenario, 20 SUs coexist with 2 randomly located PUs over $M=2$ homogeneous licensed channels, while we assume $L=3$ available power levels and $\lambda_{1}=\lambda_{2}=1$. Obviously, optimality (in terms of maximum network capacity without packet collisions) implies the activation of successive links with one vacant space. Figure 3 illustrates the MRF energy decrease during the annealing schedule and until convergence, whereas the corresponding final configuration is depicted in Figure 2. It is observed that convergence is achieved after approximately 1,000 sweeps, while the channels are intelligently allocated to protect

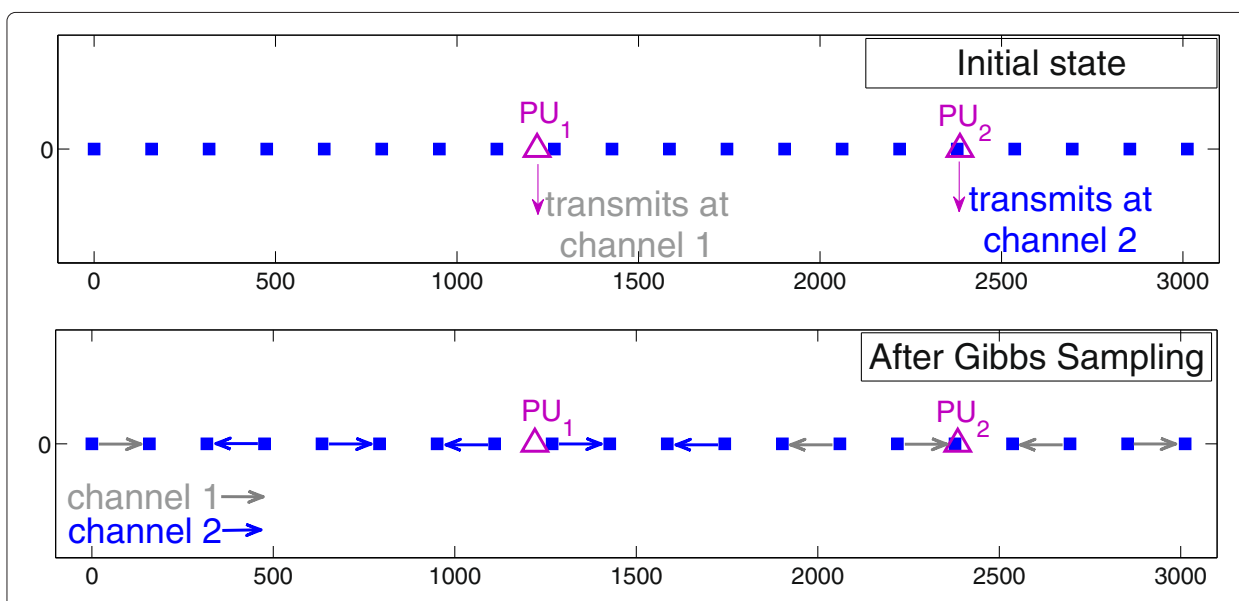

Figure 2 Examined secondary topology (before and after Gibbs sampling). 


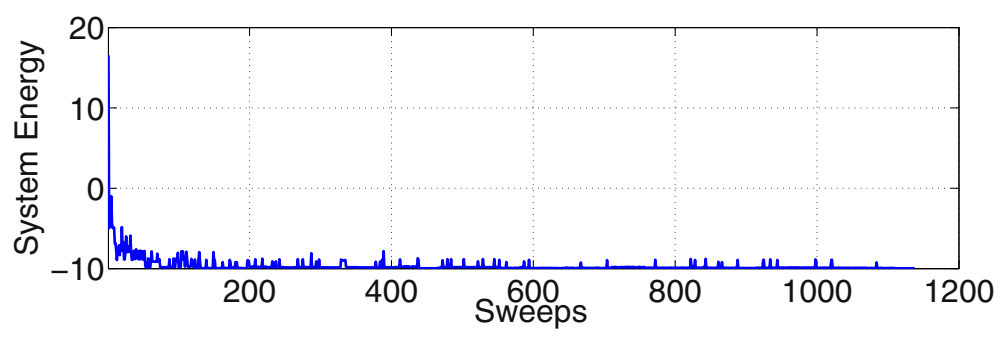

Figure 3 Convergence to minimum energy configurations (maximum value of $y$-axis represents the maximum system energy as occurred at the early sweeps).

PUs' seamless operation. We note that in the following simulated networking scenarios, if Gibbs sampling has not fully converged by 1,000 sweeps, the best solution found thus far is adopted.

\section{BPeMRF throughput optimality}

In order to compare the performance of the proposed BPeMRF scheme with the BP algorithm, we consider 14 SUs in a $2 \times 7$ grid network topology where each SU chooses another SU randomly as its destination node. Since BP does not perform power control, we consider only two power levels ( $L=2$, section 'System model') for the BPeMRF and apply the redesigned BP described in the 'Potential function via back pressure' section over 1,000 time slots. At each time slot $t$, each SU generates with probability $20 \%, n_{s}=1: 12$ packets for its corresponding destination. In Figures 4, 5, and 6, the performance of the BPeMRF in terms of the achieved capacity region, delay, and throughput respectively, is compared with BP. It is noted that in this series of experiments $\lambda_{1}$ and $\lambda_{2}$ are tuned according to Proposition 1.

Throughput (Figure 6) is expressed as the fraction of the number of packets that reached the destination divided by those sent by the source for each flow, and then the average over all flows is taken. Delay (Figure 5) is computed as the mean time difference between the packet production by the source and its arrival to the destination for each flow, while also averaged over all flows. According to the definition of the capacity region as the set of source rates for which the sum of queue lengths remains finite (section 'Potential function via back pressure'), Figure 4 provides an intuition of the set of admissible source rates, as those rates before the sum of queue lengths starts to rapidly increase (i.e., approximately up to source rate 8). From Figure 4, it can be observed that BPeMRF and the throughput

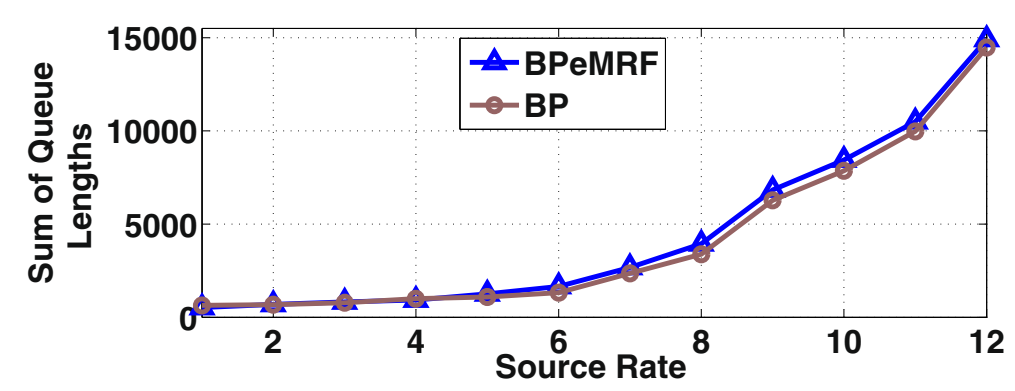

Figure 4 Sum of queue lengths (packets that have not reached their destination) by the end of the simulation. 


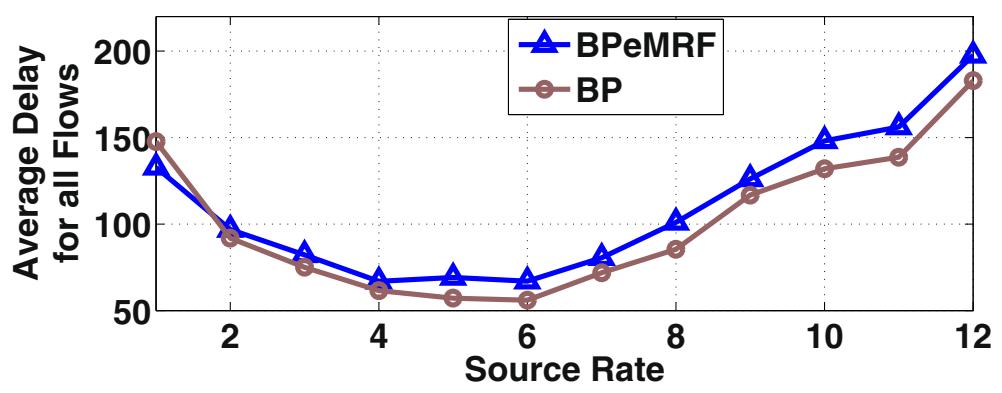

Figure 5 Average end-to-end delay for all flows (source-destination pairs).

optimal BP demonstrate very similar behavior with respect to capacity region which is in accordance with the theoretical results (Proposition 1), although BPeMRF runs for a finite number of sweeps (i.e., at most 1,000 sweeps) which implies non-perfect convergence. Therefore, BPeMRF is suitable for application in CRNs and dynamic social networks as it allows for efficient spectrum reuse and throughput optimal cross-layer network control while avoiding the NP-hard solution of the MWM. Figures 5 and 6 further reinforce the performance equivalence between BPeMRF and BP, since the induced throughput and delay values for both schemes are very close.

\section{Case study I: reputation of PUs appearance}

The main idea of the first case study is to demonstrate the benefits in CRNs whose SUs can take advantage of online social networking towards finding appropriate forwarding paths. Due to the high impact of PUs activity on secondary network performance stemmed from the requirement for ensuring seamless primary network operation - we focus on leveraging information regarding the reputation about PUs (re)appearances at specific locations with the aim of avoiding secondary routing paths across regions with high volatile PU activity. In fact, as recognized in literature [14], the dynamic nature of PUs could trigger severe cascaded secondary network modifications, thus resulting in significant reconfiguration overhead. To avoid such issues, SeBPeMRF is leveraged towards forwarding data while shaping routing paths consisting of SUs that lie in areas with low reputation for frequent PU (re)appearances.

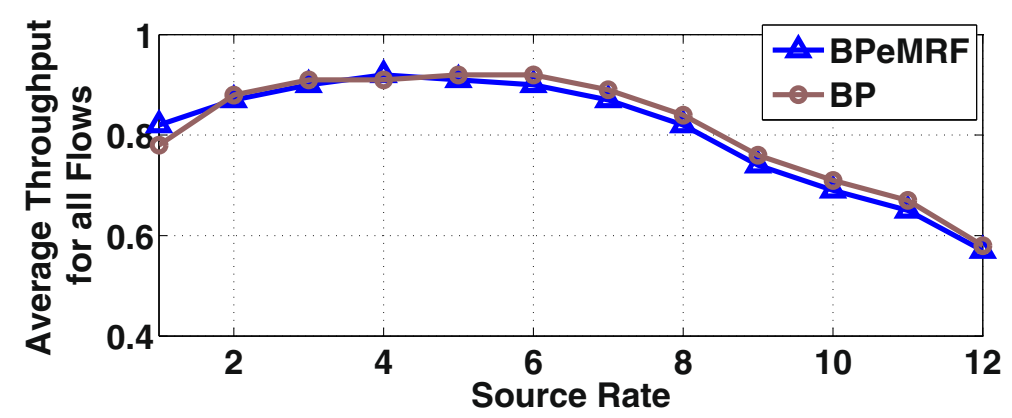

Figure 6 Average throughput for all flows given as the fraction of the packets reached the destination divided by those sent by the source. 
In the following, we adopt the model of a secondary network assisted by location-based social networking services which can help SUs to share their own experiences regarding spectrum availability in a specific position and acquire online recommendations about expected PUs appearances. For demonstration purposes, we consider $5 \times 5$ secondary grid topology in a deployment area where direct communication is only possible between one-hop neighboring nodes and $L=2$, while the reputation of PUs appearance at each location $\mathbf{z}=[x y]^{T}$ is assumed to follow a two-dimensional normal distribution $\mathcal{N}(\mu, \Sigma)$ with probability density function given by

$$
f(x, y)=\frac{1}{2 \pi \sigma_{x} \sigma_{y} \sqrt{1-\rho^{2}}} \exp \left(-\frac{1}{2}(\mathbf{z}-\mu)^{T} \Sigma^{-1}(\mathbf{z}-\mu)\right)
$$

where $\rho$ is the correlation between the corresponding variables that describe the coordinates of each location, $\mu=\left(\begin{array}{l}\mu_{x} \\ \mu_{y}\end{array}\right)$, and $\Sigma$ is the symmetric positive definite covariance matrix $\left(\begin{array}{cc}\sigma_{x}^{2} & \rho \sigma_{x} \sigma_{y} \\ \rho \sigma_{x} \sigma_{y} & \sigma_{y}^{2}\end{array}\right)$. For the examined scenario, we set the mean vector $\mu$ to correspond to the center of the deployment area and the covariance matrix $\Sigma=\left(\begin{array}{cc}1 & 0.1 \\ 0.1 & 0.5\end{array}\right)$. Figures 7 and 8 demonstrate the examined secondary topology and the associated reputation of PUs appearances, as well. We note that it is out of scope for this paper to delve into implementation issues regarding the origin of such information, which can be gained by an online social application combined with location-based services, in a distributed fashion after reaching a consensus among SUs, etc.

As proposed in the 'Exploiting social information in CRN cross-layer optimization and resource allocation' section, the weight values in parameters $\left\{\lambda_{1}, \lambda_{2}\right\}$ can effectively incorporate a variety of social relationships according to their definition. Within this framework and aiming at eliminating the anticipated disruptions caused by PU activations, we define $w(i, j, c)=w(i, j), \forall c$, by using Equation 19 such that

$$
w(i, j)=\max \left\{1+\sigma_{0} \times\left(f\left(x_{i}, y_{i}\right)-f\left(x_{j}, y_{j}\right)\right), w_{m}\right\} \forall i, j
$$

where $\left(x_{i}, y_{i}\right)$ denotes the location of $\mathrm{SU} i, \sigma_{0}$ stands for a constant value (equal to 100 for the next simulations) to control the heterogeneity of the associated weights and $w_{m}$ a

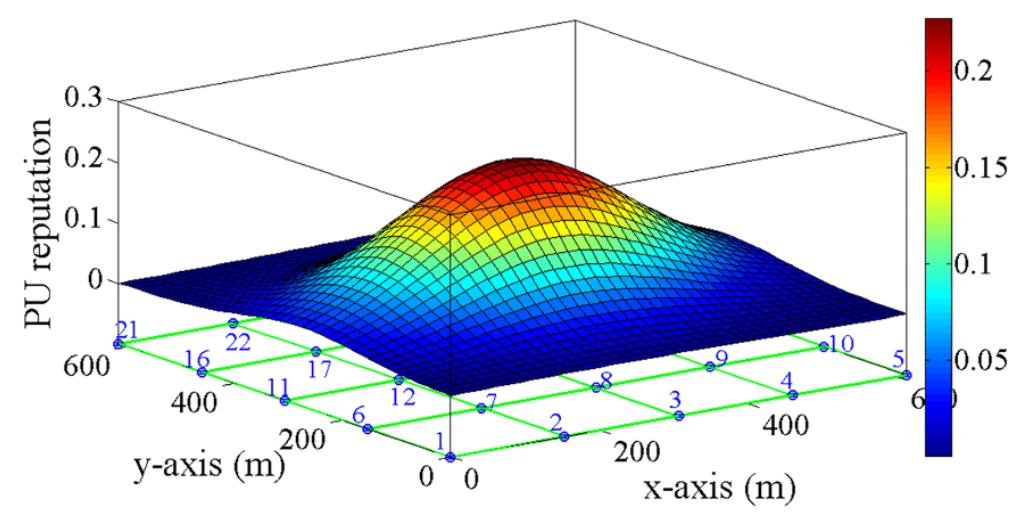

Figure 7 Examined secondary network topology and the associated reputation for PUs appearances. 


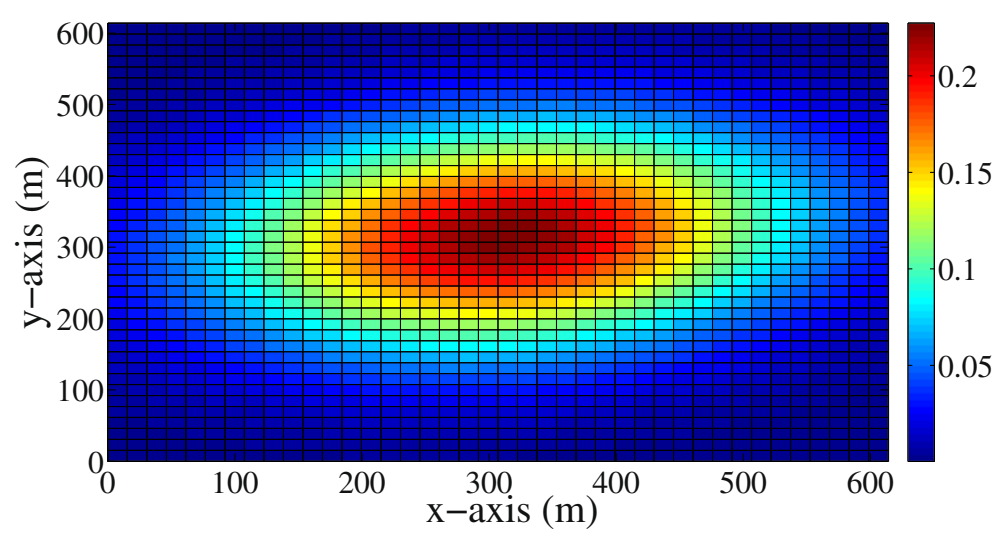

Figure 8 Two-dimensional representation of the associated reputation for PUs appearances in the examined scenario.

constant for ensuring a minimum weight value (equal to $10^{-6}$ for the examined scenario). Note that only if $j=c$, i.e., $j$ is the final destination of the commodity $c$, then $w(i, j)=$ $1+\sigma_{0} \times f\left(x_{i}, y_{i}\right)$ so as to encourage packet delivery to its corresponding destination.

Figure 9 demonstrates the secondary link activations for the examined scenario when only one active flow between SU 11 (source, located at coordinates $(1,3)$ - see also Figure 7) and SU 15 (destination, located at coordinates (5,3) - see also Figure 7) exists in the secondary network. Specifically, at each time slot $t$, the source generates - with probability $50 \%-n_{s}=10$ packets for its corresponding destination, while the simulations run over 1,000 time slots. It is observed that by utilizing weight values as defined above and leveraging the proposed SeBPeMRF framework, link activations are higher on the boundaries of the deployment area, thus prioritizing scheduling and routing on regions with lower reputation for PUs (re)appearance (Figures 7 and 8). In this manner, future disruptions caused by PU (re)activations are less probable to occur since the majority of

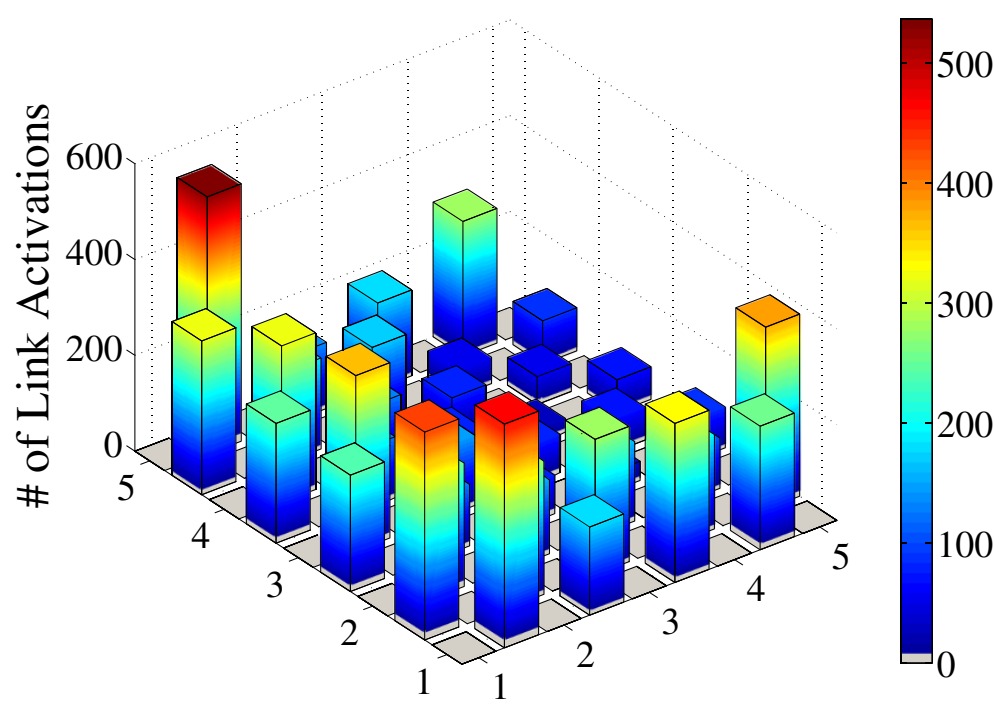

Figure 9 Secondary link activations for source-destination flow between SU 11 and SU 15. SU 11 (located at the coordinates $(1,3)$ - see also Figure 7 ) and SU 15 (located at the coordinates $(5,3)$ ). 
the data travel through forwarding paths with lower reputation for volatile primary activity and not through the shortest path, i.e., $11 \rightarrow 12 \rightarrow 13 \rightarrow 14 \rightarrow 15$, which crosses the center of the deployment area. It is noted that the number of link activations nearby the source node is higher, as it was expected, due to two main reasons. First, at the early time, slots packets have not yet arrived at the neighborhood of the destination, and thus link scheduling takes place only where queues are not empty. Secondly, due to the randomized nature of the BP-based routing, data are not always transmitted in the direction of the destination ${ }^{\mathrm{a}}$, implying longer paths and higher number of link activations near the source. On the contrary, in the locality of the destination, the number of link activations is lower since the destination operates as the network sink diminishing the randomization of the forwarding paths.

\section{Case study II: adapting routing efficiency to social layer's needs}

The second case study targets at demonstrating the benefits in a social network overlaying a CRN when the former provides feedback for the cross-layer protocol design of the latter. We assume that the traffic over the CRN is of social nature, e.g., advertisements, and also that there are no privacy issues on this traffic, i.e., the relaying nodes can decode and use themselves any information. Each relay node may segregate this traffic in two categories, namely 'useful' and 'indifferent', based on the traffic content and its interests [28]. This categorization is not strict, i.e., the nodes define probabilities that each flow's content may be useful or indifferent. Obviously, each node prefers to relay traffic assigned by itself a higher probability of being useful (based on the social profile corresponding to the node user), and thus balance the cost of forwarding by gaining through learning via preferred information.

For demonstration purposes, we consider 14 SUs in a $2 \times 7$ grid network topology with two active flows: one with source node 1 and destination node 13 (Figure 10, red circles) and the other starting from node 2 and ending at node 14 (Figure 10, blue circles). In this case study, commodities are distinguished based on their destination, i.e., there are two commodities, namely 13 and 14 . The probability that each flow's content is useful or indifferent for a specific relay is defined via the use of similarity values. Specifically, since the content of each flow should be aligned with the social profile of its destination, the aforementioned probabilities and as a result the weight values are determined as functions of the similarities of the social profiles of the relay nodes with the ones of the flows' destinations. A variety of similarity measures that can be used is listed in [29]. The similarity values used in our simulations are shown in Figure 10, unless differently mentioned, where the notation $\operatorname{Sim}(i, c)$ stands for the similarity of node $i$ with destination node $c$.

The weight on the secondary link $s=(i, j)$ corresponding to commodity $c$ takes the form:

$$
w(i, j, c)=w_{m}+\sigma_{0} \times \operatorname{Sim}(j, c) \forall i, j, c
$$

where $\sigma_{0}$ is a constant parameter used in combination with similarity values to control the heterogeneity of the weights as in Equation 20 and $w_{m}$ is a constant ensuring a minimum weight value. Note that from Equation 21, it is implied that each node $i$ should obtain knowledge about the social profiles of its one-hop neighbors and the flows' destinations. The former is direct, while for the latter, it is assumed that since the commodities are fixed $a$ priori, their destinations may have also a priori diffused in the network their 


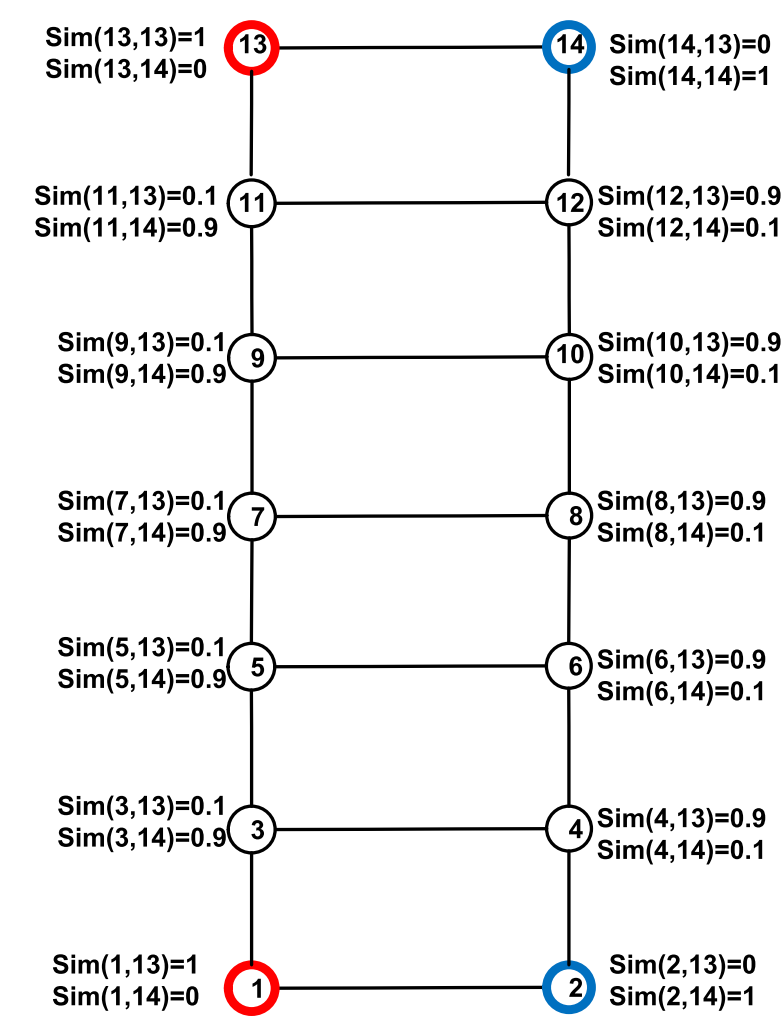

Figure 10 Topology and similarity values. There exist two flows, one from node 1 to 13 (red circles) and another from node 2 to 14 (blue circles).

social profiles. For the simulation results that follow, at each time slot, each source with probability 20\% produces a constant number of packets (ranging from 2 to 16), while each simulation runs for 500 time slots. Note that for all simulations, the links initiating from the sources are assigned the maximum possible weight value (i.e., for similarity equal to 1) for the corresponding flow so as to encourage packet releasing towards the network.

To begin with, we evaluate the improvement in the social layer's performance that can be achieved via SeBPeMRF algorithm, by comparing BPeMRF with SeBPeMRF with parameters $\sigma_{0}=10, w_{m}=1$. Note that in this case, $\frac{w_{\max }^{13}}{w_{\min }^{13}}=\frac{w_{\max }^{14}}{w_{\min }^{14}}=\frac{11}{2}$ (section 'Exploiting social information in CRN cross-layer optimization and resource allocation'). This realization of SeBPeMRF is denoted as scenario 1. The comparisons are depicted in Figures 11 and 12. The hopcount is defined as the average numbers of hops of the paths followed by the corresponding algorithm, while the path value is the average sum of weights on these paths. Obviously, for the BPeMRF algorithm, the hopcount and the path value are identical metrics, since all the weights are equal to unity. From Figure 11, it is observed that for both algorithms, packets follow paths bearing almost the same number of hops. However, the paths corresponding to the SeBPeMRF have very high path values fact that witnesses the success of SeBPeMRF in accessing the most interested relay nodes in the content of each flow. To further explain this, from Figure 10, Equation 21 and for destination 13, the links lying or being directed to the left of the topology (i.e., towards nodes $3,5,7,9,11$ ) are assigned the lowest weight value equal to 2 while the links lying or being directed to the right of the topology (i.e., towards nodes $4,6,8,10,12$ ) are 


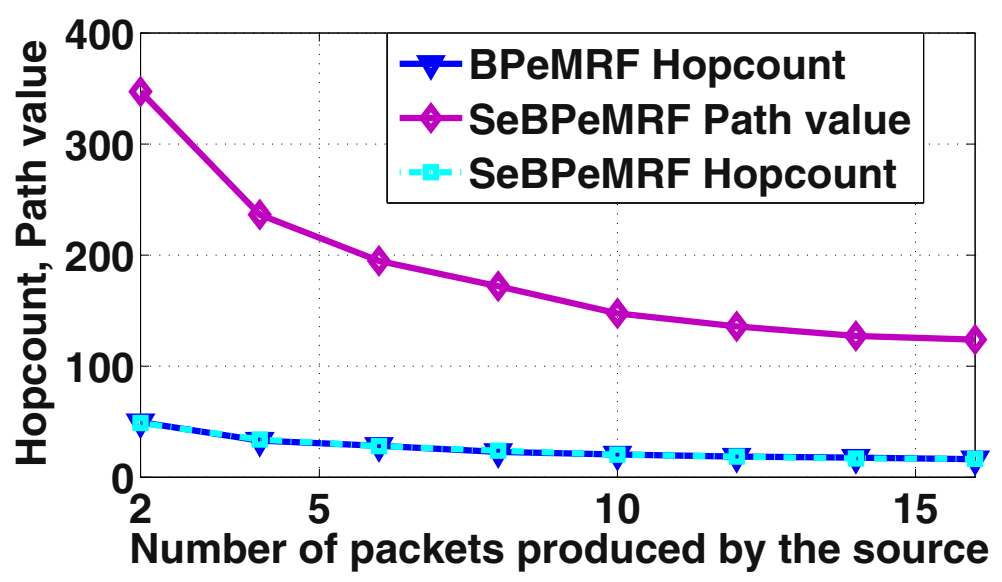

Figure 11 Comparison of the hopcount and the path value between BPeMRF and SeBPeMRF (scenario 1).

assigned the highest weight value equal to 10 (not including sources and destinations) and the opposite holds for destination 14. Then, the order of the path value compared with the hopcount (the latter is around six to seven times higher than the former for higher loads) illustrates the fact that most packets targeted to node 13 are forwarded from the right hand side of the topology and most packets targeted to node 14 are forwarded from the left hand side of the topology. Thus, SeBPeMRF tries to locate chains of interested nodes which implies that its efficiency will increase in areas where the nodes interested in a specific content are clustered, such as Figure 10. Morever, note that although hopcount initially decreases (this is due to BP's high delay under light traffic conditions, Figure 5) and then seems to be almost constant, the path value decreases as the input traffic increases due to the formula of $\lambda_{1}^{a}$ (Equation 12) which also takes into consideration the congestion via the queue backlogs. Thus, higher input traffic corresponds to higher congestion which reduces the path values due to the support of alternative links with lower weight values but with less congestion for the specific flow. Figure 12 indicates that BPeMRF and SeBPeMRF behave similarly with respect to the sum of queue lengths corresponding to packets that have not reached their destination, i.e., the loss in capacity

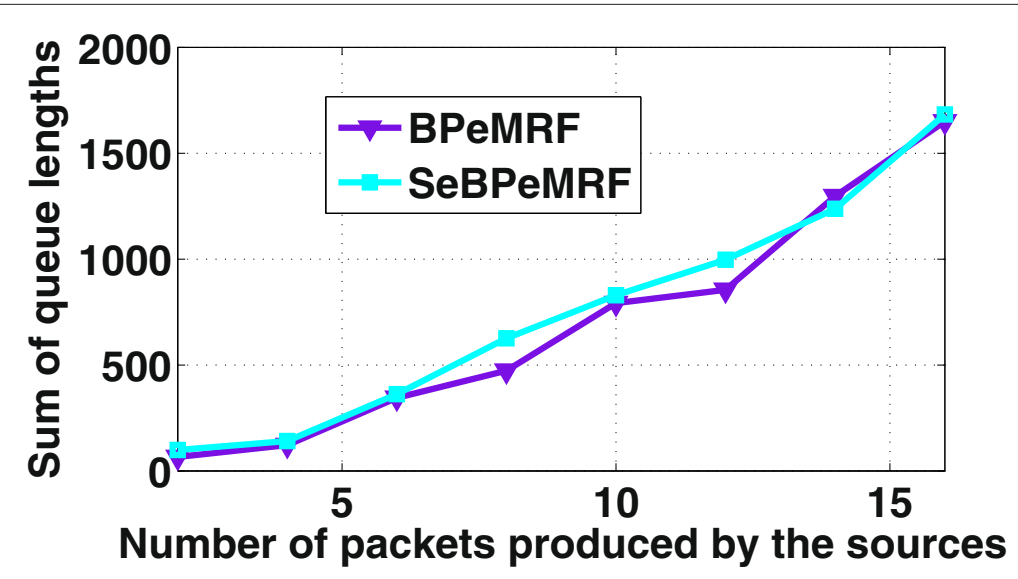

Figure 12 Comparison of the sum of queue lengths between BPeMRF and SeBPeMRF (scenario 1). 
region due to the incorporation of weight values in $\lambda_{1}$ can be considered negligible for the corresponding scenario.

In the sequel, we introduce scenario 2 for the SeBPeMRF algorithm aiming to illustrate the observation in Remark 2, based on which when $\rho^{c}$ remains constant while $w_{\max }^{c}$ and $w_{\text {min }}^{c}$ increase for a commodity $c$, then $c$ is likely to gain with respect to performance (throughput). In scenario 2, the value of $\sigma_{0}$ is different for each flow, being equal to 10 for destination 13 and 100 for destination 14. Similarly, $w_{m}$ takes the values 1 and 10 for destinations 13 and 14 correspondingly. Thus, $\frac{w_{\max }^{14}}{w_{\min }^{14}}=\frac{110}{20}=\frac{11}{2}=\frac{w_{\max }^{13}}{w_{\min }^{13}}$. Figure 13 reflects Remark 2, since it shows the tendency for higher sum of queues for destination 13 compared to the corresponding of 14 for all input rates, indicating better throughput results for the commodity with destination 14. For comparison purposes, in Figure 13, the sum of queues for each one of the commodities for scenario 1 is also depicted, where both commodities behave approximately equivalently without any tendency of one of them leading to higher queue lengths for all input rates.

Finally, the apothegm of Proposition 2 (discussed in Remark 2) is further evaluated by using the same network topology and commodities as in Figure 10 but altering the similarity values as follows. We consider that the similarities of the relay nodes with destination 13 are generated by the normal distribution with mean 0.5 and standard deviation equal to 0.01 while the similarities of the relay nodes with destination 14 follow the power-law distribution with exponent $2^{\mathrm{b}}$. Again, $\operatorname{Sim}(13,13)=1, \operatorname{Sim}(13,14)=0, \operatorname{Sim}(14,14)=$ 1 , and $\operatorname{Sim}(14,13)=0$. Thus, the weights follow the same distributions as being oneto-one functions of the similarities. Practically, according to this assignment, the weights corresponding to node 13 are well concentrated around 0.5 having a much smaller range than the ones corresponding to destination 14. In fact, for both commodities the maximum weight values remain invariant (i.e., equal to 11), while the minimum weight value is much lower for commodity 14 than 13 due to their corresponding weights' distributions. As expected from Proposition 2 and shown in Figure 14, the sum of queues remaining in the network for destination 14 is higher than the corresponding sum of 13 for all input rates, thus 13 will experience better performance in terms of throughput. Since

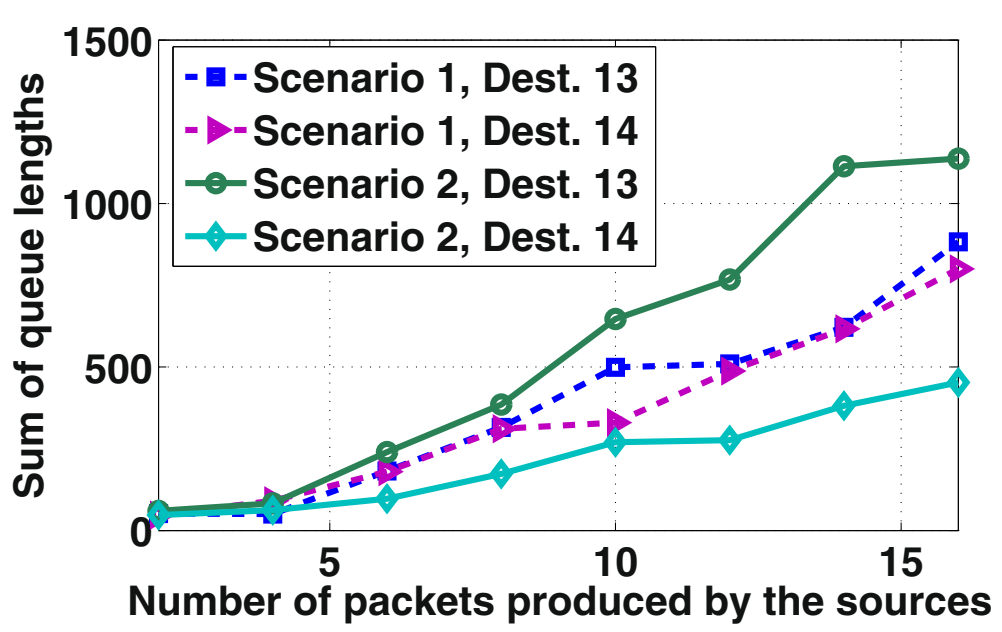

Figure 13 Comparison of the sum of queue lengths for commodities 13 and 14 for both scenarios 1 and 2. 


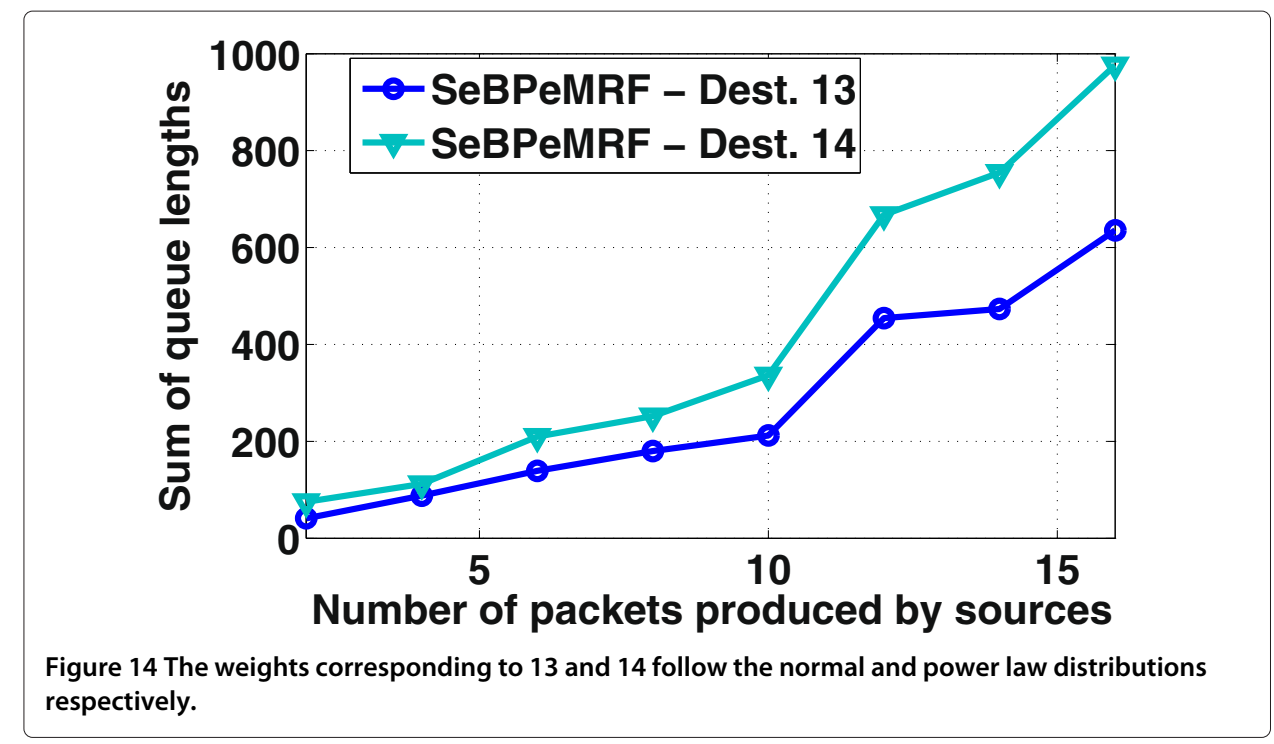

Proposition 2 captures the worst-case scenario (as also explained in Remark 2) and the latter is reflected in this example of weights' assignment, we can characterize the coexistence of power law and normally distributed similarity values/weights for two different flows as such a worst-case scenario.

\section{Conclusion}

In this paper, we studied jointly CRNs and social networking, in order to facilitate their earlier adoption in future wireless communication. We presented a BPeMRF approach for joint assignment of transmission power/channel, optimal scheduling and routing. We have demonstrated BPeMRF's efficacy in CRN infrastructures and discussed its potentials as a cross-layer approach for providing and improving QoS in various dynamic and online social network applications and services. Furthermore, we have extended BPeMRF to SeBPeMRF by incorporating social preference information available at network nodes in the resource allocation decisions and have also demonstrated its applicability and efficiency towards improving performance. Future work will be centered around extending the type of social information that can be exploited for improving network operation and performance.

\section{Endnotes}

${ }^{a}$ e.g., when a small number of packets are injected into an empty network, they may take a random walk through the network due to the lack of sufficient back pressure to drive data towards their destinations.

${ }^{\mathrm{b}}$ The values retrieved from the power law distribution are normalized so as to have maximum value equal to 1 . 
methodology and participated in the manuscript writing. VK participated in the design of the study, in the sequence alignment and drafted the manuscript. SP participated in the design of the study and its coordination.

\section{Acknowledgements}

This research is co-financed by the European Union (European Social Fund) and Hellenic national funds through the Operational Program 'Education and Lifelong Learning' (NSRF 2007-2013). E. Anifantis and E. Stai gratefully acknowledge the Foundation for Education and European Culture (IPEP) for financial support.

Received: 7 July 2014 Accepted: 9 August 2014

Published online: 15 October 2014

\section{References}

1. López-Benítez, M, Casadevall, F: Methodological aspects of spectrum occupancy evaluation in the context of cognitive radio. Eur. Trans. Telecomm. 21(8), 680-693 (2010)

2. Arkoulis, S, Anifantis, E, Karyotis, V, Papavassiliou, S, Mitrou, N: Discovering and exploiting spectrum power correlations in cognitive radio networks: an experimentally driven approach. Eur. J. Wireless Commun. Netw. 2014(1), 1-14 (2014)

3. Cisco: Cisco Visual Networking Index: Global Mobile Data Traffic Forecast Update 2013-2018. http://www.cisco. $\mathrm{com} / \mathrm{c} / \mathrm{en} / \mathrm{us} / \mathrm{solutions} /$ collateral/service-provider/visual-networking-index-vni/white_paper_c11-520862.html Accessed 22 Sept 2014

4. Chen, K-C, Chiang, M, Poor, HV: From technological networks to social networks. IEEE J. Selected Areas Commun. 31(9), 548-572 (2013)

5. Kayastha, N, Niyato, D, Wang, P, Hossain, E: Applications, architectures, and protocol design issues for mobile social networks: a survey. Proc. IEEE. 99(12), 2130-2158 (2011)

6. Haykin, S: Cognitive radio: brain-empowered wireless communications. IEEE J. Selected Areas Commun. 23(2), 201-220 (2005)

7. Karyotis, V, Stai, E, Papavassiliou, S: Evolutionary Dynamics of Complex Communications Networks. CRC Press - Taylor \& Francis Group, Boca Raton, FL, USA (2013)

8. Kindermann, R, Snell, JL: Markov Random Fields and Their Applications. American Mathematical Society, Prov., Rl, USA (1980)

9. Geman, S, Geman, D: Stochastic relaxation, gibbs distributions, and the bayesian restoration of images. IEEE Trans. Pattern Anal. Mach. Intell. 6(6), $721-741$ (1984)

10. Tassiulas, L, Ephremides, A: Stability properties of constrained queueing systems and scheduling policies for maximum throughput in multihop radio networks. IEEE Trans. Automatic Control. 37(12), 1936-1948 (1992)

11. Ofcom: TV white spaces: approach to coexistence. http://stakeholders.ofcom.org.uk/binaries/consultations/whitespace-coexistence/summary/white-spaces.pdf Accessed 22 Sept 2014

12. Hou, YT, Shi, Y, Sherali, HD: Spectrum sharing for multi-hop networking with cognitive radios. Selected Areas Commun. IEEE J. 26(1), 146-155 (2008)

13. Ding, L, Melodia, T, Batalama, SN, Matyjas, JD, Medley, MJ: Cross-layer routing and dynamic spectrum allocation in cognitive radio ad hoc networks. Vehicular Technol. IEEE Trans. 59(4), 1969-1979 (2010)

14. Arkoulis, S, Anifantis, E, Karyotis, V, Papavassiliou, S, Mitrou, N: On the optimal, fair and channel-aware cognitive radio network reconfiguration. Comput. Netw. 57(8), 1739-1757 (2013)

15. Karyotis, V, Anifantis, E, Papavassiliou, S: Cross-layer based resource management frameworks for mobile cognitive radio networks. In: Resource Management in Mobile Computing Environments. Modeling and Optimization in Science and Technologies, pp. 285-322. Springer International Publishing, Switzerland, (2014)

16. Anifantis, E, Karyotis, V, Papavassiliou, S: A markov random field framework for channel assignment in cognitive radio networks. In: 2012 IEEE International Conference on Pervasive Computing and Communications Workshops (PERCOM Workshops), pp. 770-775. IEEE, Piscataway, (2012)

17. Perreau, S, Sigelle, M, Silva, PD, Jayasuriya, A: Sensor networks protocol design using random markov field theory. In: 6th Annual IEEE Communications Society Conference on Sensor, Mesh and Ad Hoc Communications and Networks, pp. 1-8. IEEE, Piscataway, (2009)

18. Qian, LP, Zhang, YJA, Chiang, M: Distributed nonconvex power control using gibbs sampling. IEEE Trans. Commun. 60(12), 3886-3898 (2012)

19. Leconte, M, Ni, J, Srikant, R: Improved bounds on the throughput efficiency of greedy maximal scheduling in wireless networks. IEEE/ACM Trans. Netw. 19(3), 709-720 (2011)

20. Eryilmaz, A, Ozdaglar, A, Shah, D, Modiano, E: Distributed cross-layer algorithms for the optimal control of multihop wireless networks. IEEE/ACM Trans. Netw. 18(2), 638-651 (2010)

21. Chen, $\mathrm{X}$, Huang, J, Li, H: Adaptive channel recommendation for opportunistic spectrum access. IEEE Trans. Mobile Comput. 12(9), 1788-1800 (2013)

22. Li, H: Customer reviews in spectrum: recommendation system in cognitive radio networks. In: IEEE Symposium on New Frontiers in Dynamic Spectrum, pp. 1-9. IEEE, Piscataway, (2010)

23. Chen, $X$, Gong, $X$, Yang, $L$, Zhang, J: A social group utility maximization framework with applications in database assisted spectrum access. In: IEEE INFOCOM, pp. 1959-1967. IEEE, Piscataway, (2014)

24. Li, H, Huang, W, Wu, C, Li, Z, Lau, FCM: Utility-maximizing data dissemination in socially selfish cognitive radio networks. In: IEEE 8th International Conference on Mobile Adhoc and Sensor Systems (MASS), pp. 212-221. IEEE, Piscataway, (2011)

25. Stai, E, Baras, JS, Papavassiliou, S: A class of backpressure algorithms for networks embedded in hyperbolic space with controllable delay-throughput trade-off. In: Proceedings of the 15th ACM International Conference on Modeling, Analysis and Simulation of Wireless and Mobile Systems. MSWiM '12, pp. 15-22, NYC, NY, USA, (2012)

26. Iyer, A, Rosenberg, C, Karnik, A: What is the right model for wireless channel interference. IEEE Trans. Wireless Commun. 8(5), 2662-2671 (2009) 
27. Georgiadis, L, Neely, MJ, Tassiulas, L: Resource Allocation and Cross-Layer Control in Wireless Networks. Now Publishers Inc, Boston - Delft (2006)

28. Cheng, S-M, Karyotis, V, Chen, P-Y, Chen, K-C, Papavassiliou, S: Diffusion models for information dissemination dynamics in wireless complex communication networks. J. Complex Syst. 2013 (2013)

29. Cha, S-H: Comprehensive survey on distance/similarity measures between probability density functions. Int. J. Math. Models Methods Appl. Sci. 1(4), 300-307 (2007)

doi:10.1186/s40649-014-0004-1

Cite this article as: Anifantis et al.: Exploiting social features for improving cognitive radio infrastructures and social services via combined MRF and back pressure cross-layer resource allocation. Computational Social Networks 2014 1:4.

Submit your manuscript to a SpringerOpen ${ }^{\circ}$ journal and benefit from:

> Convenient online submission

- Rigorous peer review

- Immediate publication on acceptance

- Open access: articles freely available online

- High visibility within the field

- Retaining the copyright to your article

Submit your next manuscript at $\boldsymbol{\wedge}$ springeropen.com 